\title{
Disk and outflow signatures in Orion-KL: the power of high-resolution thermal infrared spectroscopy ${ }^{\star}$
}

\author{
H. Beuther, H. Linz, A. Bik, M. Goto, and Th. Henning
}

\author{
Max-Planck-Institute for Astronomy, Königstuhl 17, 69117 Heidelberg, Germany \\ e-mail: beuther@mpia-hd.mpg.de
}

Received 12 August 2009 / Accepted 4 January 2010

\begin{abstract}
Context. The Orion-KL region contains the closest examples of high-mass accretion disk candidates. Studying their properties is an essential step in studying high-mass star formation.

Aims. Resolving the molecular line emission at high spatial and spectral resolution in the immediate environment of the exciting sources to infer the physical properties of the associated gas.

Methods. We used the CRIRES high-resolution spectrograph mounted on the VLT to study the ro-vibrational ${ }^{12} \mathrm{CO},{ }^{13} \mathrm{CO}$, the Pfund $\beta$, and $\mathrm{H}_{2}$ emission between 4.59 and $4.72 \mu$ m wavelengths toward the $\mathrm{BN}$ object, the disk candidate source $\mathrm{n}$, and a proposed dust density enhancement IRC3.

Results. We detected CO absorption and emission features toward all three targets. Toward the BN object, the data partly confirm the results obtained more than 25 years ago; however, we also identify several new features. While the blue-shifted absorption is likely caused by outflowing gas, toward the $\mathrm{BN}$ object we detect $\mathrm{CO}$ in emission extending in diameter to $3300 \mathrm{AU}$ with a velocity structure close to the $v_{\mathrm{lsr}}$. Although at the observational spectral resolution limit, the ${ }^{13} \mathrm{CO}$ line width of that feature increases with energy levels, consistent with a disk origin. If one also attributes the extended $\mathrm{CO}$ emission to a disk origin, its extent is consistent with other massive disk candidates in the literature. For source n, we also find the blueshifted $\mathrm{CO}$ absorption likely from an outflow. However, it also exhibits a narrower range of redshifted $\mathrm{CO}$ absorption and adjacent weak $\mathrm{CO}$ emission, consistent with infalling motions. We do not spatially resolve the emission for source n. For both sources we conduct a Boltzmann analysis of the ${ }^{13} \mathrm{CO}$ absorption features and find temperatures between 100 and $160 \mathrm{~K}$, and $\mathrm{H}_{2}$ column densities of a few times $10^{23} \mathrm{~cm}^{-2}$. The observational signatures from IRC3 are very different with only weak absorption against a much weaker continuum source. However, the CO emission is extended and shows wedge-like position velocity signatures consistent with jet-entrainment of molecular gas, potentially associated with the Orion-KL outflow system. We also present and discuss the Pfund $\beta$ and $\mathrm{H}_{2}$ emission in the region.

Conclusions. This analysis toward the closest high-mass disk candidates outlines the power of high spectral and spatial resolution mid-infrared spectroscopy for studying the gas properties close to young massive stars. We will extend qualitatively similar studies to larger samples of high-mass young stellar objects to constrain the physical properties of the dense innermost gas structures in more detail and in a statistical sense.
\end{abstract}

Key words. stars: formation - stars: early-type - accretion, accretion disks - techniques: spectroscopic - ISM: jets and outflows stars: individual: Orion-BN, Orion source n

\section{Introduction}

Understanding the physical structure of massive accretion disks is one of the main unsolved problems in high-mass star formation. Although indirect, the main line of arguments for accretion disks stems from massive molecular outflow observations that identify collimated and energetic outflows from high-mass young stellar objects (YSOs, e.g., Henning et al. 2000; Beuther et al. 2002b; Zhang et al. 2005; Arce et al. 2007). Collimated jet-like outflow structures are usually attributed to massive accretion disks and magneto-centrifugal acceleration. Recent 2D and 3D magneto-hydrodynamical simulations of massive collapsing gas cores also result in the formation of massive accretion disks (Yorke \& Sonnhalter 2002; Krumholz et al. 2007, 2009). However, it is still unclear whether such massive disks are similar to their low-mass counterparts, hence dominated by the central YSO and in Keplerian rotation, or whether they are maybe self-gravitating non-Keplerian entities.

\footnotetext{
* Based on observations of the ESO program 380.C-0380(A).
}

While studies at (sub)mm wavelengths are a powerful tool to mainly study the cold gas and dust components on spatial scales on the order of $1000 \mathrm{AU}$ (e.g., Cesaroni et al. 2007), such observations are not that well suited to investigate the inner and warmer components of massive rotating structures. In contrast to that, mid-infrared spectral lines, e.g., ro-vibrationally excited $\mathrm{CO}$ emission lines, can trace these warm gas components. However, the spectral and/or spatial resolution was mostly lacking because absorption features were dominating, hence prohibiting the detection of the accretion disks in emission. Several recent studies have further demonstrated the power of high-spectral and high-spatial resolution CO mid-infrared spectroscopy for disks around low-mass YSOs and Herbig Ae stars (e.g., Goto et al. 2006; Pontoppidan et al. 2008; van der Plas et al. 2009).

To achieve the highest angular and spectral resolution possible, we observed some of the closest massive disk candidates in Orion at a distance of 414 pc (Menten et al. 2007) - Orion-BN (the Becklin-Neugebauer Object), source n and IRC3 - in the 
Table 1. Source positions (from Dougados et al. 1993).

\begin{tabular}{lrr}
\hline \hline Source & $\begin{array}{r}\text { RA } \\
(\mathrm{J} 2000.0)\end{array}$ & $\begin{array}{r}\text { Dec. } \\
(\mathrm{J} 2000.0)\end{array}$ \\
\hline BN object & $05 \mathrm{~h} 35 \mathrm{~m} 14.12 \mathrm{~s}$ & $-05 \mathrm{~d} 22 \mathrm{~m} 22.9 \mathrm{~s}$ \\
Source n & $05 \mathrm{~h} 35 \mathrm{~m} 14.35 \mathrm{~s}$ & $-05 \mathrm{~d} 22 \mathrm{~m} 32.9 \mathrm{~s}$ \\
IRC3 & $05 \mathrm{~h} 35 \mathrm{~m} 13.90 \mathrm{~s}$ & $-05 \mathrm{~d} 22 \mathrm{~m} 30.0 \mathrm{~s}$ \\
\hline
\end{tabular}

$\mathrm{CO} v=1-0$ transitions around $4.65 \mu \mathrm{m}$ with the CRyogenic high resolution InfraRed Echelle Spectrograph (CRIRES, Käufl et al. 2004) at the VLT. Both objects are well detected at mid-infrared wavelengths exhibiting various kinds of disk-signatures.

The Becklin-Neugebauer Object: since its detection in the 1960s, the BN object has been one of the archetypical highmass YSOs (Becklin \& Neugebauer 1967; Henning et al. 1990). Scoville et al. (1983) observed the source in several frequency settings between 2 and $5 \mu \mathrm{m}$ and detected molecular emission from several $\mathrm{CO}$ isotopologues (fundamental and overtone emission), and they inferred that BN exhibits an outflow/wind, as well as a highly confined region of molecular gas at high densities and temperatures of $\sim 3500 \mathrm{~K}$. The estimated luminosity of the BN object is $1-2 \times 10^{4} L_{\odot}$ corresponding to a B0.5 main sequence star (Scoville et al. 1983). More recently, Jiang et al. (2005) observed $\mathrm{BN}$ in polarized near-infrared emission and identified signatures caused by an embedded accretion disk. The BN object has a high velocity along the line of sight of $\sim 21 \mathrm{~km} \mathrm{~s}^{-1}$ compared with the cloud velocity of around $5 \mathrm{~km} \mathrm{~s}^{-1}$ (Scoville et al. 1993). This is consistent with the measured high proper motions of that object (e.g., Plambeck et al. 1995). Whether the BN object is expelled from the Trapezium system or during a disintegration of a bound system once containing source I, source $\mathrm{n}$ and the $\mathrm{BN}$ object itself is still a matter of debate (e.g., Tan 2004; Gómez et al. 2005; Zapata et al. 2009).

Source n: based on a bipolar radio morphology and $\mathrm{H}_{2} \mathrm{O}$ maser association, Menten \& Reid (1995) suggested that this source may be one of the driving sources of the powerful molecular outflows within Orion-KL. Extended mid-infrared emission was observed perpendicular to the outflow axis (Greenhill et al. 2004; Shuping et al. 2004), and Luhman (2000) detected CO overtone emission. Both features are interpreted as likely being due to an accretion disk. Source $\mathrm{n}$ is believed to be in an evolutionary younger stage than the BN object, and the luminosity is estimated to be lower as well, on the order of $2000 L_{\odot}$ (Greenhill et al. 2004).

IRC3: the source $\mathrm{n}$ observations serendipitously covered the extended infrared source IRC3 (e.g., Dougados et al. 1993) which we present here as well. At $3.6 \mu \mathrm{m}$ wavelengths, IRC3 is elongated in the northeast-southwest direction (Dougados et al. 1993) and shows highly polarized near- to mid-infrared emission (Minchin et al. 1991). The observations are consistent with IRC3 being a dust density enhancement reprocessing light from another source, potentially IRC2 (Downes et al. 1981; Minchin et al. 1991; Dougados et al. 1993).

Figure 1 gives an overview of the region marking the sources discussed in the paper as well as the slit orientations (see also Sect. 2). The nominal absolute positions for the three sources are listed in Table 1.

\section{Observations}

We obtained high-resolution spectra between 4.6 and $4.7 \mu \mathrm{m}$ with CRIRES (Käufl et al. 2004) mounted on UT1 at the VLT on Paranal, Chile. Two grating settings were selected (12/-1/n,

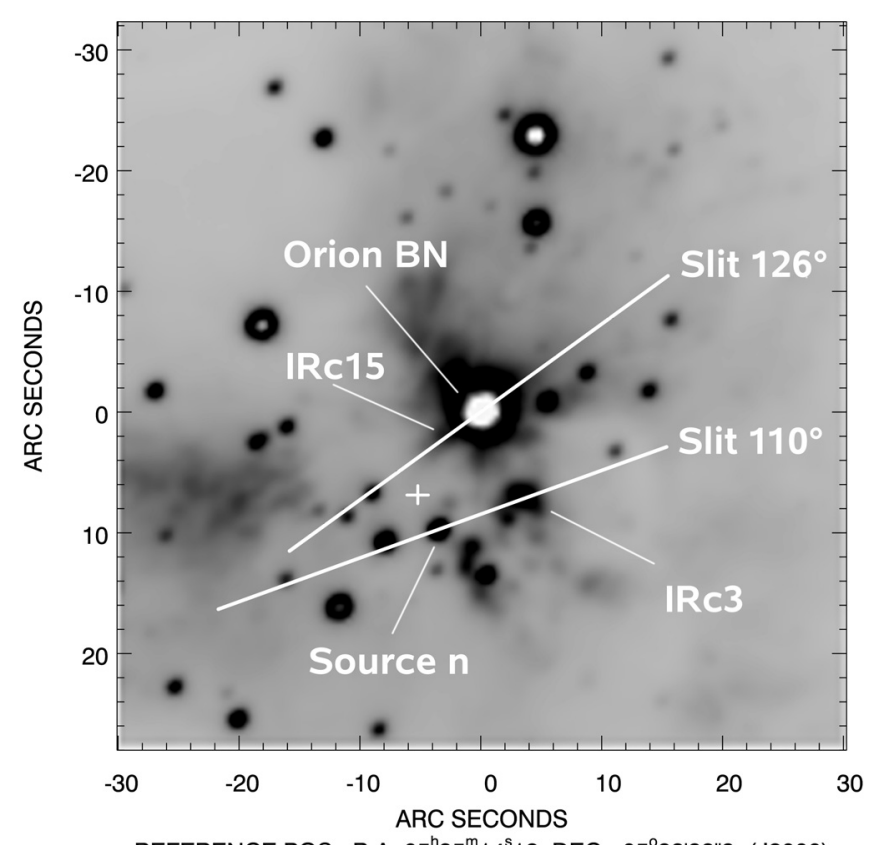

REFERENCE POS. R.A. $05^{\mathrm{h}} 35^{\mathrm{m}} 14^{\mathrm{s}} \cdot 12$ DEC $-05^{\circ} 22^{\prime} 22$ ".9 (J2000)

Fig. 1. Overview image of the region. The grey-scale $K_{\mathrm{s}}$ band image is taken from UKIDSS DR5 (Lawrence et al. 2007), the white stellar peak positions are due to saturation. The slits and important sources are marked. The cross denotes the position of IRc2.

$\lambda_{\text {ref }}=4662.1$ and $\left.12 /-1 / \mathrm{i}, \lambda_{\text {ref }}=4676.1\right)$ to observe the spectral interval covering the ${ }^{12} \mathrm{CO} v=1-0[\mathrm{P}(1)-\mathrm{P}(5) / \mathrm{R}(0)-\mathrm{R}(8)]$ lines without gaps.

One slit covered the BN object with a position angle of 126 degrees east of north, whereas the second slit included source $n$ and IRC 3 with a position angle of 110 degrees east of north. For the strong source BN, only 40 secs $($ DIT $=2$ secs, NDIT $=10$ ) were required. For the second object with the weaker sources we had a total on-slit integration time of $20 \mathrm{~min}$ (DIT $=10 \mathrm{secs}$, NDIT =2). For the BN observation a slit width of $0.2^{\prime \prime}$ was used while for the observation of source $n$ a slit with of $0.4^{\prime \prime}$ was selected which correspond to spectral resolving powers $\lambda / \Delta \lambda$ of 100000 and 50000 , respectively. The non-AO mode was applied since no natural guide star is available in the close environment. The infrared seeing measured from the spectra was $0.35^{\prime \prime}$ during the BN observation and $0.45^{\prime \prime}$ for the source $n$ observation. The nod-throw of all the observations was set to $10^{\prime \prime}$. To correct for the telluric absorption lines, attached to every science observation a telluric standard star (HR 1666 with spectral type A3III) was observed.

As extended emission in the $\mathrm{CO}$ lines was present in the observations, we corrected the frames for distortion in order to get a wavelength solution valid for the whole chip. Firstly, the chips are slightly rotated with respect to the slit (ranging from 0.05 degree for chip 3 to 0.45 degree in the case of chip 4 ). We measured the position of the brightest object as function of wavelength and calculate from the displacement in position the rotation angle. Secondly, after the rotation angle has been corrected, the curvature of the slit is corrected by measuring the position (central wavelength) of a sky-line as function of the spatial coordinate. This could be described with a 2 nd degree polynomial. Using the IDL routines polywarp and poly_2d, the distortion was corrected.

After the distortion correction, the raw files are processed by the ESO CRIRES pipeline (version 1.10.1) in combination with 
the Gasgano software. The data are dark subtracted, flat field corrected as well as corrected for non-linearity. The wavelength calibration is done using the telluric emission lines in combination with a HITRAN model spectrum (Rothman et al. 2005). A crosscorrelation of the spectra with the HITRAN spectra showed that the wavelength accuracy of the spectra is $\pm 0.5 \mathrm{~km} \mathrm{~s}^{-1}$.

Two absorption lines are present in the standard star: the Pfund $\beta$ and the Humphreys $\epsilon$ line. Due to the strong telluric absorption, these lines are not trivial to remove. Therefore, we first reduced the spectrum without correcting for the intrinsic absorption lines of the standard star. In the final reduced spectrum these lines become eminent as emission lines free from contamination by the atmosphere. The Pfund $\beta$ line is also seen in our science object. However, the Humphreys $\epsilon$ line is not present in the science spectrum of $\mathrm{BN}$ before division by the standard star. Therefore, it can be used to correct for the absorption lines of the standard star. We used a high resolution Kurucz model spectrum from an A0V star (http://kurucz. harvard.edu/stars.html) and scaled and shifted the model spectrum such that the Humphreys $\epsilon$ profile would fit that of the observed Humphreys $\epsilon$ profile in the standard star. Assuming that the Pfund $\beta$ line scales in the same way as the Humphreys $\epsilon$ line does, we divided with the model spectrum to remove the line contamination.

The spectra were corrected for the earth velocity to the local standard of rest using rvcorrect in IRAF. The velocity corrections applied for the two observing dates were $-3.8 \mathrm{~km} \mathrm{~s}^{-1}$ for the BN data (observed on 21st October 2007) and $+43.2 \mathrm{~km} \mathrm{~s}^{-1}$ for the source n/IRC3 observations (taken on 21st February 2008). The velocity relative to the local standard of rest $v_{\mathrm{lsr}}$ of Orion varies between 2.5 and $9 \mathrm{~km} \mathrm{~s}^{-1}$ (e.g., Comito et al. 2005), and we adopt the approximate value of $+5 \mathrm{~km} \mathrm{~s}^{-1}$.

\section{Results}

Toward all three sources we detected the whole suite of ${ }^{12} \mathrm{CO} v=$ $1-0$ lines present in the spectral window, the ${ }^{13} \mathrm{CO} v=1-0$ lines from $\mathrm{R}(6)$ to $\mathrm{R}(13)$ that were not blended by the ${ }^{12} \mathrm{CO} v=$ $1-0$ lines, as well as the Pfund $\beta$ line. Figure 2 presents the complete spectrum toward the BN object, and Table 2 gives an overview of the covered lines, their wavelengths $\lambda$ and the lowerlevel energy state of the transitions $\left(E_{\text {lower }} / k\right)$. In total, this setup covers a broad range of energy levels extending up to $504 \mathrm{~K}$.

\subsection{The BN object}

\subsubsection{CO absorption and emission}

Figure 3 presents a zoom compilation of ${ }^{12} \mathrm{CO}$ data from the $\mathrm{R}(1)$ to $\mathrm{R}(8)$ line covering lower energy levels between 5.5 and $199 \mathrm{~K}$. If one ignores the telluric line feature at $\sim 3 \mathrm{~km} \mathrm{~s}^{-1}$ two broad absorption features can be identified at approximately -14 and $+8 \mathrm{~km} \mathrm{~s}^{-1}$. Since all ${ }^{12} \mathrm{CO}$ lines are saturated, their peak absorption velocities are unreliable, and we refer to the ${ }^{13} \mathrm{CO}$ data (Fig. 4). The peak velocity of the blue-shifted component is $\sim-15 \mathrm{~km} \mathrm{~s}^{-1}$ extending from $\sim-28$ to $\sim-8 \mathrm{~km} \mathrm{~s}^{-1}$. The second absorption feature has its peak at $\sim 8 \mathrm{~km} \mathrm{~s}^{-1}$, close to the $v_{\text {lsr }}$ of the cloud, and extends from $\sim-8$ to $\sim 18 \mathrm{~km} \mathrm{~s}^{-1}$. Furthermore, red-shifted from the absorption we clearly identify a $\mathrm{CO}$ emission peaking in ${ }^{12} \mathrm{CO}$ and ${ }^{13} \mathrm{CO}$ at $\sim 20 \mathrm{~km} \mathrm{~s}^{-1}$ and extending from $\sim 15$ to $\sim 30 \mathrm{~km} \mathrm{~s}^{-1}$. The overall extent of the ${ }^{12} \mathrm{CO}$ absorption and emission is from $\sim-30$ to $\sim+30 \mathrm{~km} \mathrm{~s}^{-1}$. It should be noted that while the $v_{\text {lsr }}$ of the different cloud components for the Orion-KL region vary between approximately 3
Table 2. Observed lines.

\begin{tabular}{lrr}
\hline \hline Line & $\lambda$ & $E_{\text {lower }} / k$ \\
& $\mu \mathrm{m}$ & $\mathrm{K}$ \\
\hline${ }^{12} \mathrm{CO}$ & & \\
$\mathrm{R}(9)$ & 4.5876 & 249 \\
$\mathrm{R}(8)$ & 4.5950 & 199 \\
$\mathrm{R}(7)$ & 4.6024 & 155 \\
$\mathrm{R}(6)$ & 4.6090 & 116 \\
$\mathrm{R}(5)$ & 4.6176 & 83 \\
$\mathrm{R}(4)$ & 4.6254 & 55 \\
$\mathrm{R}(3)$ & 4.6333 & 33 \\
$\mathrm{R}(2)$ & 4.6412 & 17 \\
$\mathrm{R}(1)$ & 4.6493 & 5.5 \\
$\mathrm{R}(0)$ & 4.6575 & 0 \\
$\mathrm{P}(1)$ & 4.6742 & 5.5 \\
$\mathrm{P}(2)$ & 4.6826 & 17 \\
$\mathrm{P}(3)$ & 4.6912 & 33 \\
$\mathrm{P}(4)$ & 4.7000 & 55 \\
$\mathrm{P}(5)$ & 4.7088 & 83 \\
\hline${ }^{13} \mathrm{CO}$ & & \\
$\mathrm{R}(13)$ & 4.6641 & 504 \\
$\mathrm{R}(12)$ & 4.6741 & 432 \\
$\mathrm{R}(11)$ & 4.6782 & 365 \\
$\mathrm{R}(10)$ & 4.6853 & 304 \\
$\mathrm{R}(9)$ & 4.6926 & 249 \\
$\mathrm{R}(8)$ & 4.7000 & 199 \\
$\mathrm{R}(7)$ & 4.7075 & 155 \\
$\mathrm{R}(6)$ & 4.7150 & 116 \\
\hline $\mathrm{Pfund} \beta$ & 4.6538 & $157954^{a}$ \\
$\mathrm{H}(0-0 \mathrm{~S} 9$ & 4.6947 & $7198^{a}$ \\
\hline & &
\end{tabular}

Notes. ${ }^{(a)}$ The temperatures listed for the Pfund $\beta$ and $\mathrm{H}_{2} 0-0 \mathrm{~S} 9$ lines do not indicate gas temperatures since these lines are not excited by collisions within thermal gas, but rather by ionizing photons and within shocked regions, respectively.

and $9 \mathrm{~km} \mathrm{~s}^{-1}$, Scoville et al. (1983) inferred that the corresponding velocity of the $\mathrm{BN}$ object is significantly higher around $21 \mathrm{~km} \mathrm{~s}^{-1}$ (consistent with the different BN ejection scenarios, e.g., Tan 2004; Gómez et al. 2005). While the absorption features stem from the warmer protostellar envelopes and the surrounding cloud with usual temperatures on the order of $100 \mathrm{~K}$ (see also Boltzmann analysis below), the ro-vibrational lines in emission can be caused by different processes. For example, fluorescence via UV photons or resonance scattering from strong infrared fields can excite these lines without significantly heating the gas (e.g., Blake \& Boogert 2004; Ryde \& Schöier 2001). Alternatively, the ro-vibrational lines could be caused by hotter gas components (see discussion in Sect. 4). We note that the critical densities of these lines are on the order of $10^{13} \mathrm{~cm}^{-3}$ which practically implies that extended gas components can be hardly responsible for the emission.

Although the blue-shifted part of the spectrum with respect to the $v_{\text {lsr }}$ of the molecular cloud is slightly broader than the redshifted part, nevertheless we clearly identify red-shifted absorption as well. To first order, the blue-shifted gas seen in absorption can be identified with outflowing gas from the region, whereas the red-shifted features belong to gas infalling in the direction of the central source. The outflowing gas with a maximum velocity relative to the $v_{\text {lsr }}$ of $\sim 35 \mathrm{~km} \mathrm{~s}^{-1}$ is consistent with outflow wings often observed at $\mathrm{mm}$ wavelengths from young massive 


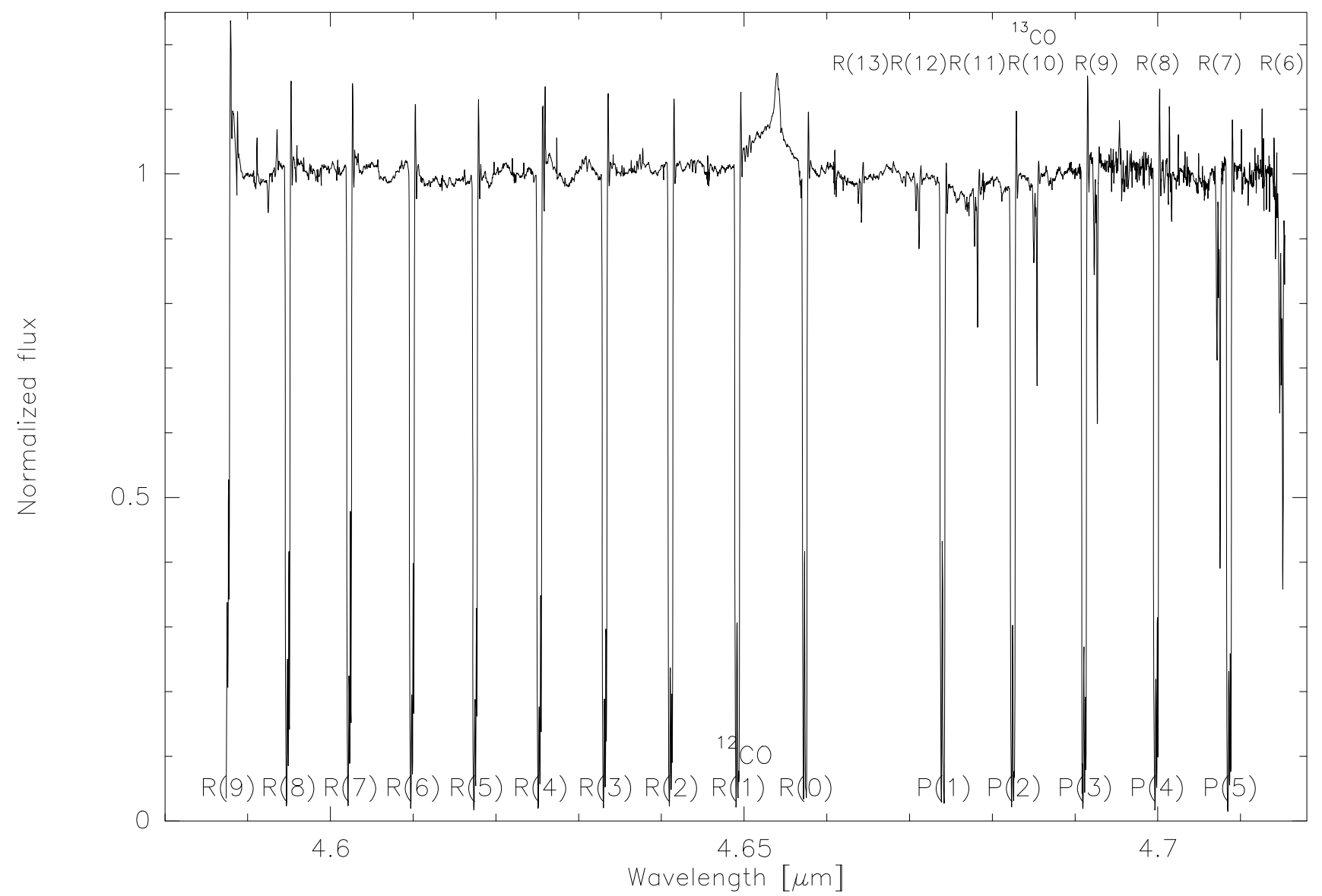

Fig. 2. CRIRES observations of the R and P CO line series around $4.65 \mu \mathrm{m}$. The strong absorption features are the CO lines, and the emission line at $\sim 4.654 \mu \mathrm{m}$ is Pfund $\beta$.

star-forming regions (e.g., Beuther et al. 2002b). It should be noted that the even broader outflow wings observed at mm wavelength toward Orion-KL exceeding $\pm 50 \mathrm{~km} \mathrm{~s}^{-1}$ (e.g., Chernin \& Wright 1996) are likely not related to the BN object but rather to one or more sources about $10^{\prime \prime}$ south-east of BN (source I, source $\mathrm{n}$ and/or SMA1, e.g., Greenhill et al. 2004; Jiang et al. 2005; Bally 2008; Beuther \& Nissen 2008).

How do these general features compare with the data published by Scoville et al. (1983) which were observed during several observing runs between December 1977 and February 1981 , hence about 30 years prior to our observations. The general CO line structure with two strong absorption features plus one red-shifted emission peak are largely the same. Also the overall extent of the emission is quite similar. Compared to our measured values of $\sim-15, \sim+9$ and $\sim 20 \mathrm{~km} \mathrm{~s}^{-1}$ for the three components, Scoville et al. (1983) report for the corresponding features velocities of $-18,+9$ and $+20 \mathrm{~km} \mathrm{~s}^{-1}$. While two velocities agree well, the most blue-shifted absorption peak appears to have shifted a little bit between the two observations. However, given that their spectral resolution was more than a factor 2 lower than that of the new CRIRES data $\left(7\right.$ versus $\left.3 \mathrm{~km} \mathrm{~s}^{-1}\right)$, we refrain from further interpretation of this. Furthermore, Scoville et al. (1983) identify two more absorption features, one at $-3 \mathrm{~km} \mathrm{~s}^{-1}$ and one at $+30 \mathrm{~km} \mathrm{~s}^{-1}$. Regarding the $-3 \mathrm{~km} \mathrm{~s}^{-1}$ component we are not able to infer any changes because that features lies very close to the telluric emission which obscures any reliable signature there. However, Fig. 3 shows that we do not detect any additional absorption feature blue-shifted from the $26.6 \mathrm{~km} \mathrm{~s}^{-1}$ emission. Therefore, the $+30 \mathrm{~km} \mathrm{~s}^{-1}$ absorption dip reported by
Scoville et al. (1983) was either a transient feature or not significant with respect to the signal-to-noise ratio.

Since the ${ }^{12} \mathrm{CO}$ data are so strongly saturated, for the following analysis we use the corresponding ${ }^{13} \mathrm{CO}$ data covering the R(6) to R(13) transitions (Fig. 2). Since the absorption depth $I / I_{0}$ is related to the optical depth $\tau$ via $\frac{I}{I_{0}}=\mathrm{e}^{-\tau}$ we can directly estimate the optical depth of the ${ }^{13} \mathrm{CO}$ absorption lines shown in Fig. 4 if the lines are spectrally resolved. Since the full width at zero intensity (FWZI) is on the order of $20 \mathrm{~km} \mathrm{~s}^{-1}$ (see above), this criterion is fulfilled with our spectral resolution $\lambda / \Delta \lambda=10^{5}$ which corresponds to a velocity resolution of $3 \mathrm{~km} \mathrm{~s}^{-1}$. Except of the lowest ${ }^{13} \mathrm{CO} \mathrm{R}(6)$ line component at $+9 \mathrm{~km} \mathrm{~s}^{-1}$ all other observed ${ }^{13} \mathrm{CO}$ absorption features have optical depths below 1 . This allows us to estimate rotational temperatures via Boltzmann plots from the equivalent line width following the approach outlined in Scoville et al. (1983) also adopting their finite optical depth corrections. Using their Eq. (A8), the column density $N_{l}$ of the lower-level energy state is

$N_{l}=1.13 \times 10^{12} \frac{F A}{f_{\mathrm{lu}}} \mathrm{cm}^{-2}$

with $F, A$ and $f_{\text {ul }}$ denoting the optical depth correction following the appendix in Scoville et al. (1983), the equivalent line width (in units of $\mathrm{cm}^{-1}$ ) and absorption oscillator strength, respectively. Because the absorption feature at $\sim 9 \mathrm{~km} \mathrm{~s}^{-1}$ is affected by the telluric line, in the following we only work with the data from the $\sim-15 \mathrm{~km} \mathrm{~s}^{-1}$ line. The parameters and the calculated column densities are listed in Table 3. In a Boltzmann 


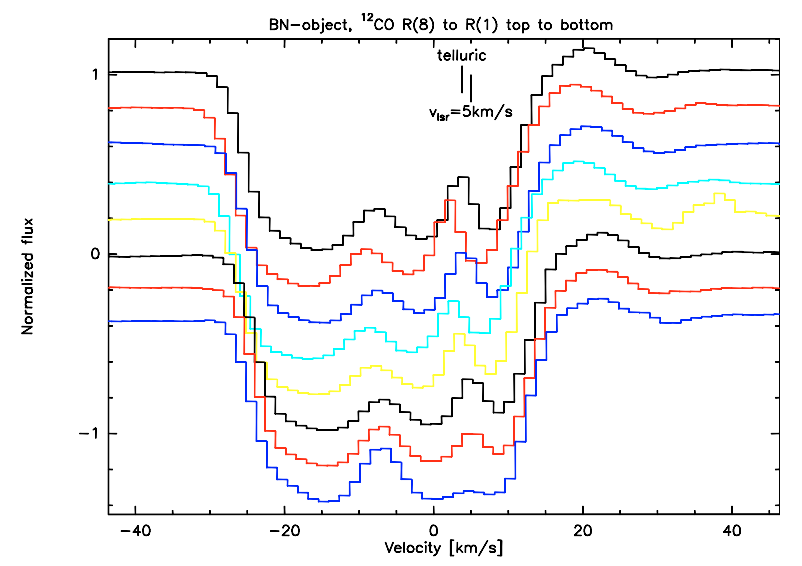

Fig. 3. The ${ }^{12} \mathrm{CO} \mathrm{R}(8)$ to $\mathrm{R}(1)$ lines from top to bottom toward the $\mathrm{BN}$ object. Telluric and $v_{\text {lsr }}$ velocities are marked. The feature between 0 and $8 \mathrm{~km} \mathrm{~s}^{-1}$ is a telluric artifact.

Table 3. ${ }^{13} \mathrm{CO}$ values for Boltzmann plots.

\begin{tabular}{|c|c|c|c|c|c|c|}
\hline & $\begin{array}{r}E_{\text {lower }} / k \\
\mathrm{~K}\end{array}$ & $\begin{array}{r}A \\
\mathrm{~km} \mathrm{~s}^{-1}\end{array}$ & $\begin{array}{r}f_{\mathrm{ul}} \\
\times 10^{-6}\end{array}$ & $F$ & $g_{J}$ & $\begin{array}{r}N_{l}\left({ }^{13} \mathrm{CO}\right) \\
\times 10^{15} \mathrm{~cm}^{-2}\end{array}$ \\
\hline \multicolumn{7}{|c|}{$\mathrm{BN} @-15 \mathrm{~km} \mathrm{~s}^{-1}$} \\
\hline $\mathrm{R}(6)$ & 116 & 3.70 & 6.0 & 1.18 & 13 & 6.0 \\
\hline$R(7)$ & 155 & 3.16 & 5.9 & 1.14 & 15 & 5.0 \\
\hline $\mathrm{R}(9)$ & 249 & 1.90 & 5.9 & 1.08 & 19 & 2.9 \\
\hline $\mathrm{R}(10)$ & 304 & 1.36 & 5.8 & 1.06 & 21 & 2.0 \\
\hline $\mathrm{R}(11)$ & 365 & 0.69 & 5.8 & 1.04 & 23 & 1.0 \\
\hline $\mathrm{R}(12)$ & 432 & 0.51 & 5.8 & 1.02 & 25 & 0.7 \\
\hline $\mathrm{R}(13)$ & 504 & 0.27 & 5.8 & 1.02 & 27 & 0.4 \\
\hline \multicolumn{7}{|c|}{ source n@-7 km s s-1 comp. } \\
\hline $\mathrm{R}(7)$ & 155 & 4.1 & 5.9 & 1.14 & 15 & 6.5 \\
\hline $\mathrm{R}(9)$ & 249 & 2.7 & 5.9 & 1.08 & 19 & 4.1 \\
\hline $\mathrm{R}(10)$ & 304 & 2.0 & 5.8 & 1.08 & 21 & 3.1 \\
\hline $\mathrm{R}(12)$ & 432 & 1.3 & 5.8 & 1.06 & 25 & 2.0 \\
\hline $\mathrm{R}(13)$ & 504 & 1.5 & 5.8 & 1.05 & 27 & 2.2 \\
\hline \multicolumn{7}{|c|}{ source n @ $+5 \mathrm{~km} \mathrm{~s}^{-1}$ comp. } \\
\hline $\mathrm{R}(7)$ & 155 & 4.6 & 5.9 & 1.23 & 15 & 7.8 \\
\hline $\mathrm{R}(9)$ & 249 & 2.7 & 5.9 & 1.16 & 19 & 4.4 \\
\hline $\mathrm{R}(10)$ & 304 & 1.8 & 5.8 & 1.12 & 21 & 2.8 \\
\hline $\mathrm{R}(12)$ & 432 & 0.6 & 5.8 & 1.04 & 25 & 0.9 \\
\hline $\mathrm{R}(13)$ & 504 & 0.1 & 5.8 & 1.00 & 27 & 0.1 \\
\hline
\end{tabular}

Notes. The table entries are explained in the main text.

distribution, the total column density $N_{\text {tot }}\left({ }^{13} \mathrm{CO}\right)$ is related to the temperature and the lower state column density via:

$\ln \left(\frac{N_{l}}{g_{l}}\right)=\ln \left(\frac{N_{\text {tot }}\left({ }^{13} \mathrm{CO}\right)}{Q(T)}\right)-\frac{1}{T_{\text {rot }}} \frac{E_{\text {lower }}}{k}$

with $g_{l}$ the statistical weight of the lower-level transition and $Q(T)$ the partition function at the given temperature.

Figure 5 presents the corresponding Boltzmann plot where the lower-state column density $N_{l}$ divided by the statistical weight $g_{J}$ is plotted against the lower-state energy $E_{\text {lower }}$ divided by $k$. A linear fit to the data gives the rotational temperature $T_{\text {rot }}$ and the column density of ${ }^{13} \mathrm{CO}$ divided by the partition function $Q(T)$. For the $-15 \mathrm{~km} \mathrm{~s}^{-1}{ }^{13} \mathrm{CO}$ component the fitted $T_{\text {rot }}$ is $\sim 112 \pm 20 \mathrm{~K}$. Scoville et al. (1983) calculated the rotation temperature for the more blue-shifted absorption component from the ${ }^{12} \mathrm{CO} v=2-0$ transitions, and they find $\sim 150 \mathrm{~K}$ there, which is approximately consistent with our new determination. The other fitted parameter is $N_{\text {tot }}\left({ }^{13} \mathrm{CO}\right) / \mathrm{Q}(\mathrm{T}) \approx 1.35 \times 10^{15} \mathrm{~cm}^{-2}$. For 2-atomic molecules like ${ }^{13} \mathrm{CO}$ the partition function can be approximated by $Q(T=112 \mathrm{~K}) \sim \mathrm{kT} /(\mathrm{hB}) \sim 42$ (where $B$ is the rotation constant), and we get a ${ }^{13} \mathrm{CO}$ column density of $\sim 5.7 \times 10^{16} \mathrm{~cm}^{-2}$. Using furthermore the ${ }^{12} \mathrm{CO}$ to ${ }^{13} \mathrm{CO}$ isotopologic ratio of 69 (e.g., Sheffer et al. 2007), we derive a total CO column density of the $-15 \mathrm{~km} \mathrm{~s}^{-1}$ component toward Orion-BN of $\approx 3.9 \times 10^{18} \mathrm{~cm}^{-2}$. These column density estimates are in excellent agreement with the results derived by Scoville et al. (1983) from the ${ }^{12} \mathrm{CO} v=2-0$ lines.

How do these values compare to other observations? The $\mathrm{BN}$ object was detected at $1.3 \mathrm{~mm}$ wavelength by Blake et al. (1996) at a $0.15 \mathrm{Jy}$ level with a spatial resolution of $1.5^{\prime \prime} \times 1.0^{\prime \prime}$. Following Plambeck et al. (1995), about $50 \mathrm{mJy}$ of that flux can be attributed to circumstellar dust emission. Assuming optically thin dust emission, an average dust temperature of $100 \mathrm{~K}$, a dust opacity index $\beta$ of 2 and a standard gas-to-dust ratio of 100 , we can calculate the total $\mathrm{H}_{2}$ column density (Hildebrand 1983; Ossenkopf \& Henning 1994; Henning et al. 1995; Beuther et al. 2002a, 2005a; Draine et al. 2007). The derived $\mathrm{H}_{2}$ column density and mass within their $1.5^{\prime \prime} \times 1.0^{\prime \prime}$ synthesized beam are then $\sim 1.3 \times 10^{24} \mathrm{~cm}^{-2}$ and $0.1 M_{\odot}$, respectively. For comparison, using a standard CO-to- $\mathrm{H}_{2}$ ratio of $8 \times 10^{-5}$, the $\mathrm{H}_{2}$ column density estimated here from the CO IR spectroscopy data is $\sim 4.9 \times 10^{22} \mathrm{~cm}^{-2}$. While the mm continuum data trace all velocity components along the line of sight, the near-infrared data trace selected velocity components. Judging from Figs. 3 and 4, where we see more than 1 velocity component, the total column density traced by the $\mathrm{CO}$ data is more than a factor 2 higher. Furthermore, the $\mathrm{CO}$ absorption only traces the gas in the foreground of the near-infrared source reducing the traced gas by another factor 2 . While both approaches are affected by systematics - e.g., the mm derived column densities can be wrong by a factor 5 depending on the assumptions on the dust properties and temperatures - other reasons are more important for some of the differences. In particular, the mm continuum emission is sensitive to the cold and warm dust emission whereas the nearinfrared absorption of the ${ }^{13} \mathrm{CO}$ transitions useable for our analysis traces mainly the warmer gas. Therefore, these data indicate that a large fraction of the gas is at relatively low temperatures.

\subsubsection{Potential signatures from the BN disk?}

While most of the observed emission is spatially unresolved, we find weak extended ${ }^{12} \mathrm{CO}$ emission toward the $\mathrm{BN}$ object. Figure 6 presents a position-velocity diagram along the slit direction (PA of $126^{\circ}$ east of north, see Sect. 2) which corresponds to the proposed disk orientation (Jiang et al. 2005). We find CO emission around the rest velocity of the BN object of $\sim 21 \mathrm{~km} \mathrm{~s}^{-1}$ extending approximately \pm 4 " in both directions. Since we do not identify any strong velocity dispersion, this extended emission is unlikely to be due to an outflow, but it may potentially come from the proposed disk (Jiang et al. 2005). The measured extent of $\sim 8^{\prime \prime}$ would then correspond at the given distance of Orion of $414 \mathrm{pc}$ to an approximate disk diameter of $\sim 3300 \mathrm{AU}$ or a disk radius of $1650 \mathrm{AU}$. While such disk size would be comparably large with respect to typical low-mass disks (e.g., several reviews in Reipurth et al. 2007), it is consistent with measured sizes of rotating structures in other high-mass star-forming regions (e.g., Schreyer et al. 2002; Cesaroni et al. 2005; Beltrán et al. 2006; Beuther \& Walsh 2008).

While we do not identify extended emission is the rarer ${ }^{13} \mathrm{CO}$ isotopologue, the $\mathrm{CO}$ and ${ }^{13} \mathrm{CO}$ emission feature around $20 \mathrm{~km} \mathrm{~s}^{-1}$ exhibits an additional interesting feature in the ${ }^{13} \mathrm{CO}$ 


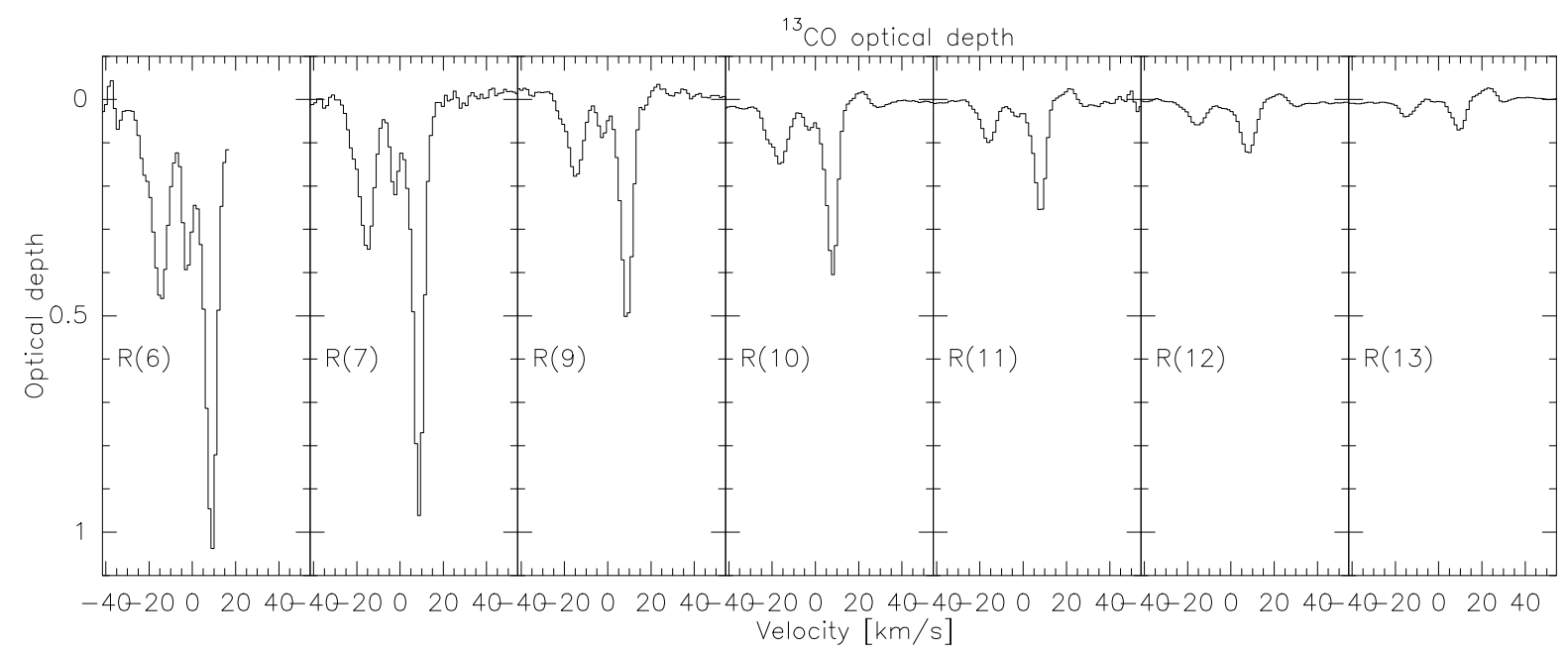

Fig. 4. ${ }^{13} \mathrm{CO}$ optical depths toward the BN object.

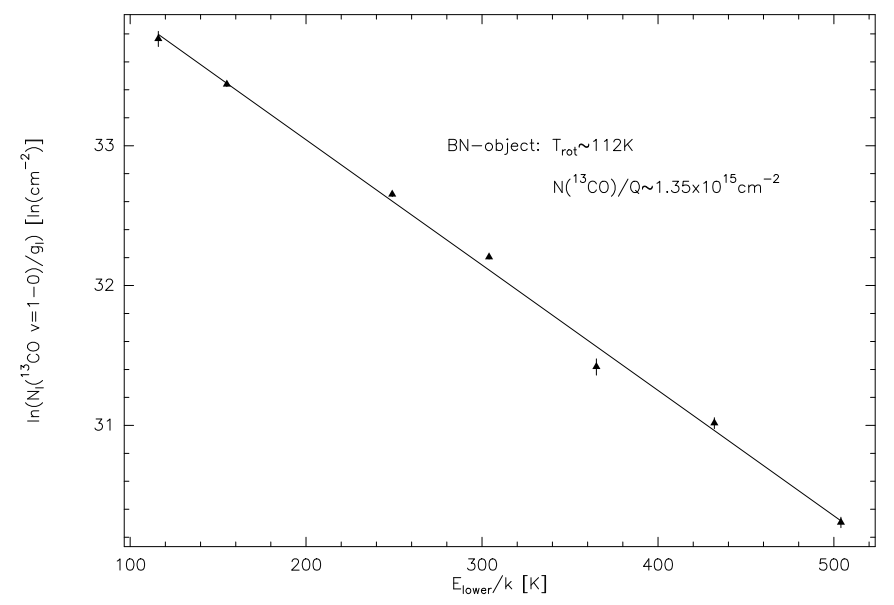

Fig. 5. Boltzmann plot for the ${ }^{13} \mathrm{CO} v=1-0$ lines toward the $\mathrm{BN}$ object. The $x$-axis shows the lower-level energies of the transitions and the $y$-axis presents the natural logarithm of the corresponding column densities divided by their statistical weights.

data (Fig. 4): while the emission feature is at the edge of the bandpass for the ${ }^{13} \mathrm{CO} \mathrm{R}(6)$ line, it remains undetected for the $\mathrm{R}(7)$ line and only comes up for the lines greater $\mathrm{R}(9)$. This emission feature is also visible in all ${ }^{12} \mathrm{CO}$ lines $(\mathrm{R}(1)$ to $\mathrm{R}(8))$ shown in Fig. 3, but it does not exhibit any significant shape change there. Likely, the ${ }^{12} \mathrm{CO}$ emission is optically thick and traces only an outer envelope that does not show big variations with excitation temperature. In contrast to that, the measured Gaussian line width of that component for the more optically thin ${ }^{13} \mathrm{CO} \mathrm{R}(9)$, $\mathrm{R}(10), \mathrm{R}(11), \mathrm{R}(12)$ and $\mathrm{R}(13)$ lines are 4.3, 5.3, 6.5, 6.4 and $6.6 \mathrm{~km} \mathrm{~s}^{-1}$, respectively. Although errors on the line width are difficult to quantify because the emission is at the flank of the strong absorption feature, and furthermore our spectral resolution is only $3 \mathrm{~km} \mathrm{~s}^{-1}$, the data are indicative of a line width increase with excitation temperature of the line that appears to saturate for the highest detectable transitions $\mathrm{R}(11)$ to $\mathrm{R}(13)$. While for a centrifugally supported disk with a central mass of $\sim 10 M_{\odot}$ the circular velocity at $3300 \mathrm{AU}$ is relatively low on the order of $\sim 1.6 \mathrm{~km} \mathrm{~s}^{-1}$, the measured line width increase is consistent with a rotating disk structure because the inner region with higher rotation velocities should have higher temperatures caused by the exciting central star. One has to keep in mind that for purely thermal excitation, rotation would not cause such an effect because

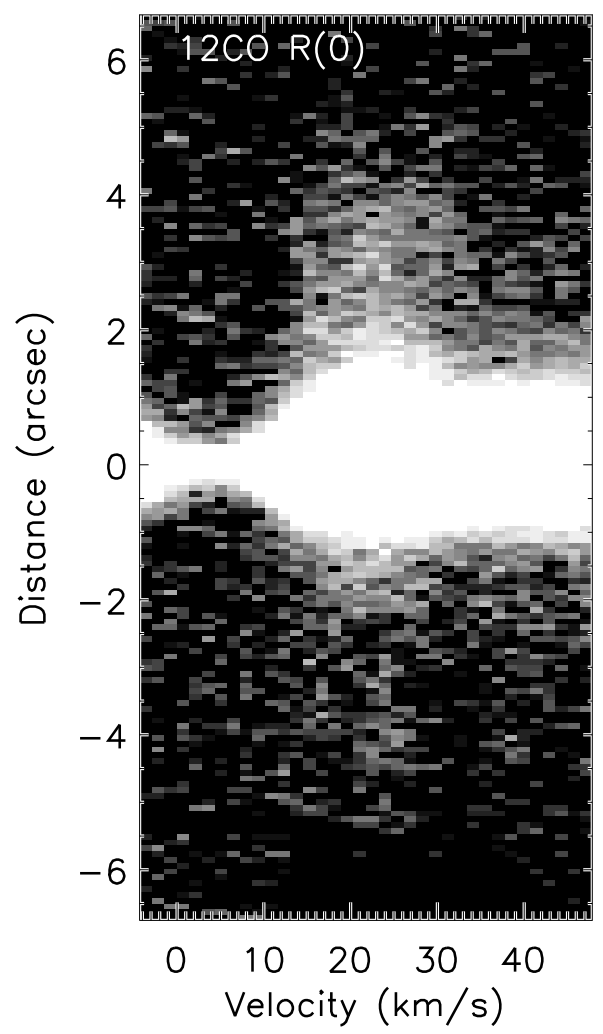

Fig. 6. Position velocity diagram of the ${ }^{12} \mathrm{CO}$ (R0) line toward the $\mathrm{BN}$ object along the slit direction (PA of $126^{\circ}$ east of north, see Sect. 2) plotted in logarithmic intensity scale. Around offset 0 the figure is dominated by the continuum emission, but outside $\sim \pm 2$ " the emission stems from the CO line. Positive offsets go in northwest direction.

the upper levels - all in the $3000 \mathrm{~K}$ regime - do not exhibit big relative excitation differences. Hence, they do not trace significantly different regions of a disk then. In contrast to that, for lines that are excited by UV fluorescence such an effect is possible because the excitation mechanism via an electronic excited state tends to preserve the level population of the $v=0$ rotational states (e.g., Brittain et al. 2009). Therefore, in this case the R(9) to $R(13)$ lines are sensitive to gas temperatures in a relatively broader range between 150 and $500 \mathrm{~K}$ (Table 2). An exact reproduction of the line width increase would require a detailed disk 


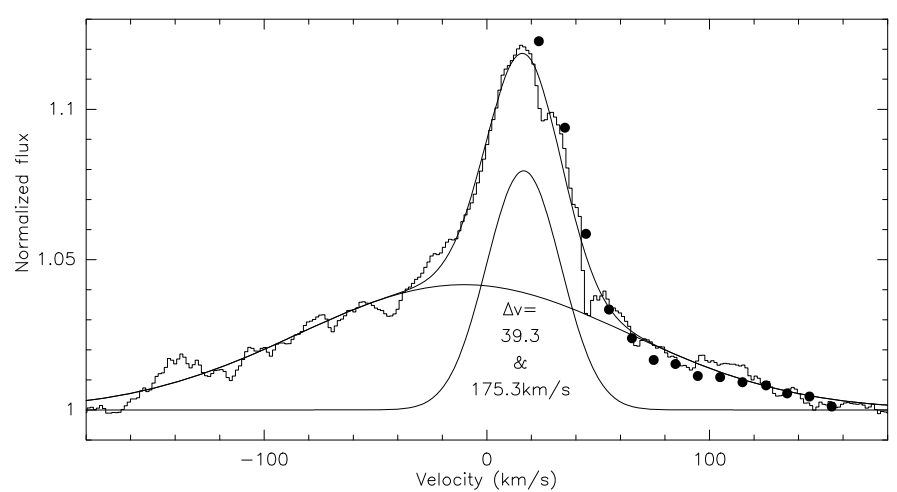

Fig. 7. Hydrogen recombination Pfund $\beta$ line toward the BN object. The histogram shows the data, and the three other lines present a twocomponent Gaussian fit to the data. Both Gaussians are shown separately as well as the combined fit. The FWHM $\Delta v$ of both components is given in the figure. The big dots reproduce the best fit to the $\mathrm{Br} \alpha$ line (their Fig. 7 re-scaled to our normalized Pfund $\beta$ spectrum) for an optically thin outflow with a velocity law $v \propto r^{-2 / 3}$ by Scoville et al. (1983).

model, including its density and temperature structure. Since we cannot constrain these parameters well from our data, this is beyond the scope of this paper. Although the line width increase is below our nominal spectral resolution element, qualitatively the observations are consistent with a rotating disk structure.

\subsubsection{The Pfund $\beta$ line toward the $\mathrm{BN}$ object and $\mathrm{H}_{2}$ emission nearby $\mathrm{BN}$}

Figure 7 presents a zoom into the Pfund $\beta$ line. The line shape consists of two components, one central Gaussian component and broad line wings. It is possible to fit the whole profile relatively well with a two-component Gaussian fit where the central component has a $F W H M \Delta v=39.3 \mathrm{~km} \mathrm{~s}^{-1}$ and the broad component has a $F W H M$ of $\Delta v=175.5 \mathrm{~km} \mathrm{~s}^{-1}$. The full width down to zero intensity is approximately $340 \mathrm{~km} \mathrm{~s}^{-1}$ (between -170 and $170 \mathrm{~km} \mathrm{~s}^{-1}$ ). Close to the peak of the profile at around $25 \mathrm{~km} \mathrm{~s}^{-1}$, the spectrum exhibits a small dip which is likely an artifact from the telluric corrections (see Sect. 2). Considering this, the peak of the central Gaussian fit at $\sim 16.5 \mathrm{~km} \mathrm{~s}^{-1}$ is still consistent with the velocity derived for the BN object by Scoville et al. (1983) of $\sim 21 \mathrm{~km} \mathrm{~s}^{-1}$.

Figure 7 also presents as thick dots the best fit obtained by Scoville et al. (1983) for the $\operatorname{Br} \alpha$ line (their Fig. 7 re-scaled to our normalized spectrum). Their model consists of a supersonic, optically thin decelerating outflow with a velocity law of $v \propto r^{-2 / 3}$. It is remarkable how well the shape of their $\operatorname{Br} \alpha$ line obtained $\sim 30$ years ago corresponds to the shape of the newly observed Pfund $\beta$ line. Hence, these Pfund $\beta$ data are also consistent with their outflow model.

Furthermore, approximately 4 " south-east of the BN object we detect $\mathrm{H}_{2}$ emission from the $\mathrm{H}_{2} \mathrm{O}-0 \mathrm{~S} 9$ transition with $E_{\text {lower }} / k=7198 \mathrm{~K}$. This $\mathrm{H}_{2}$ emission feature is spatially associated with the corresponding $\mathrm{H}_{2}$ knot in near-infrared $\mathrm{H}_{2}$ images (e.g., Nissen et al. 2007) as well as with the mid-infrared source IRc15 reported by Shuping et al. (2004). Figure 8 shows a position velocity diagram of this feature. While we do not resolve any spatial structure of the $\mathrm{H}_{2}$ knot, it shows a very broad velocity extent going to blue-shifted velocities of $\sim-80 \mathrm{~km} \mathrm{~s}^{-1}$, in excess of the $\mathrm{CO}$ absorption features measured toward the $\mathrm{BN}$ object. Since we are mainly interested in the BN object, source $n$

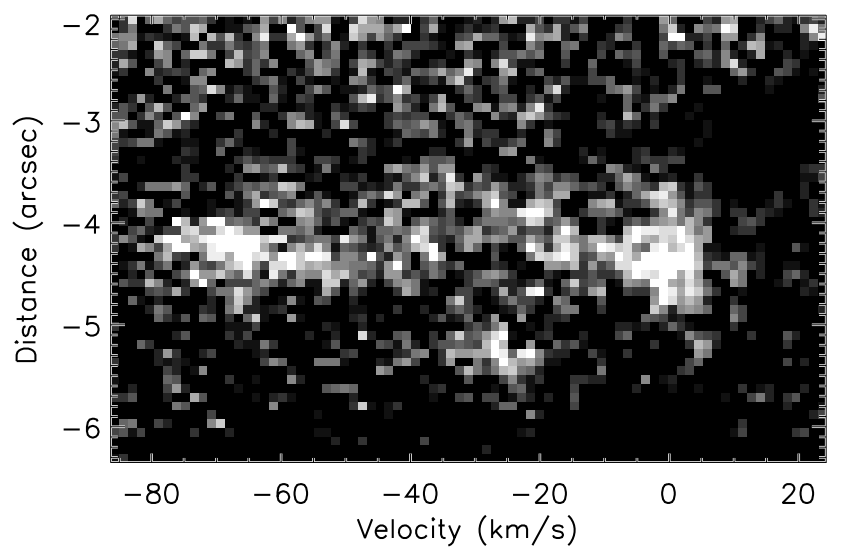

Fig. 8. Position velocity digram of the $\mathrm{H}_{2}$ emission 4.3" south-east of the BN-object associated with the infrared source IRC15 (Dougados et al. 1993).

and IRC3, we refrain from further analysis of this offset $\mathrm{H}_{2}$ emission.

\subsection{Source $n$}

\subsubsection{CO absorption and emission}

Due to the different observing dates, for source n (and IRC3 in the following section) the telluric lines are almost entirely shifted out of the $\mathrm{CO}$ spectrum. Figures 9 and 10 present the corresponding ${ }^{12} \mathrm{CO} \mathrm{R}$-series lines and the detected ${ }^{13} \mathrm{CO}$ lines with their associated optical depths. In comparison to the previous BN data, for source $\mathrm{n}$ we only identify one broad absorption feature in the ${ }^{12} \mathrm{CO}$ data which is dominated by blue-shifted outflowing gas. It is interesting to note that the most blue-shifted absorption does not have a Gaussian shape but rather a more extended wing-like structure. In the framework of accelerated winds with distance from the driving star or disk, such a spectral behavior would be expected. These accelerated winds increase in velocity with distance from the star. Simultaneously, with increasing distance from the driving source the density of the surrounding gas and dust envelope also decreases, lowering the corresponding absorption depth at those velocities. Hence, in this picture, higher-velocity gas should show shallower absorption features in the spectra (e.g., Lamers \& Cassinelli 1999). Furthermore, we also identify a red-shifted emission component at $\sim+15 \mathrm{~km} \mathrm{~s}^{-1}$. However, compared to the BN object, where the emission feature can be identified in all transitions, for source $\mathrm{n}$ it is more prominent in the higher excited lines. The total width of the $\mathrm{CO}$ absorption and emission is $\sim 90 \mathrm{~km} \mathrm{~s}^{-1}$ ranging from approximately $\sim-65$ to $\sim+25 \mathrm{~km} \mathrm{~s}^{-1}$ (broader than for BN). The blue-shifted end is less well determined because of telluric line contamination. Nevertheless the blue-shifted outflow part of the spectrum extends $\sim 70 \mathrm{~km} \mathrm{~s}^{-1}$ from the assumed $v_{\mathrm{lsr}}$ of $+5 \mathrm{~km} \mathrm{~s}^{-1}$. This value exceeds that measured toward BN by about $20 \mathrm{~km} \mathrm{~s}^{-1}$. Although at the edge of the telluric line contamination, we tentatively identify an additional discrete absorption feature at $\sim-35 \mathrm{~km} \mathrm{~s}^{-1}$.

Since the ${ }^{12} \mathrm{CO}$ line is again always saturated (Fig. 9), for the additional analysis we use the corresponding ${ }^{13} \mathrm{CO}$ data (Fig. 10). Because of line-blending and telluric emission, we only measure five ${ }^{13} \mathrm{CO}$ lines for source $n$. The broad, saturated ${ }^{12} \mathrm{CO}$ absorption feature is clearly resolved into two components with approximate velocities at $\sim-7 \mathrm{~km} \mathrm{~s}^{-1}$ and $\sim+5 \mathrm{~km} \mathrm{~s}^{-1}$. While the $\sim+5 \mathrm{~km} \mathrm{~s}^{-1}$ shows the deeper absorption features for 


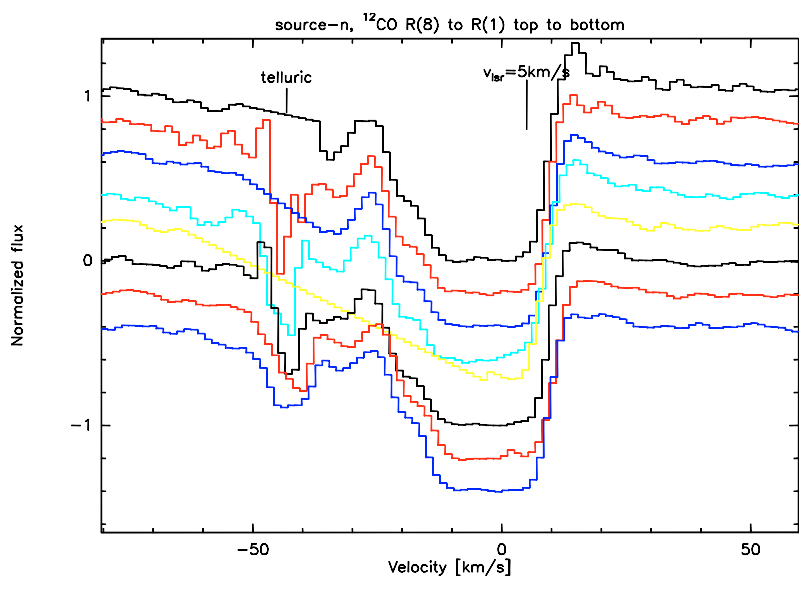

Fig. 9. The ${ }^{12} \mathrm{CO} \mathrm{R}(8)$ to $\mathrm{R}(1)$ lines from top to bottom toward source n. Telluric and $v_{\mathrm{lsr}}$ velocities are marked. The linear slopes and dips around $-40 \mathrm{~km} \mathrm{~s}^{-1}$ associated with the $\mathrm{R}(8), \mathrm{R}(6)$ and $\mathrm{R}(4)$ lines (for the latter extending from $\sim-5$ to $\sim-65 \mathrm{~km} \mathrm{~s}^{-1}$ ) are artifacts due to the telluric corrections.

the lower energy R-lines, it is interesting to note that the single absorption peak observed for the ${ }^{13} \mathrm{CO} \mathrm{R}(13)$ line is associated with the $\sim-7 \mathrm{~km} \mathrm{~s}^{-1}$ component, indicating hotter gas at more blue-shifted velocities. Following the approach outlined for BN in Sect. 3.1.1, the derived optical depth of ${ }^{13} \mathrm{CO}$ is $\sim 0.6$ at its highest.

Since the telluric lines are far away in velocity space (see Fig. 9), for source $n$ we can conduct the Boltzmann analysis for both absorption components separately. The measured equivalent widths $A$ for both components are listed in Table $3^{1}$. Again calculating the ${ }^{13} \mathrm{CO} v=1$ column densities (Table 3 ) and producing Boltzmann plots (Fig. 11), we find that for both velocity components fits to all 5 data points are less good than in the case of the BN object. This is likely due to the fact that fitting 2 Gaussians to the broad $\mathrm{R}(13)$ line, which does not show two well separated absorption features anymore, may overestimate the contribution of the $\sim-7 \mathrm{~km} \mathrm{~s}^{-1}$ component and hence underestimate the contribution from the $\sim+5 \mathrm{~km} \mathrm{~s}^{-1}$ component. Therefore, we also fit only the 4 lower-energy transitions between $\mathrm{R}(7)$ and $\mathrm{R}(12)$ resulting in better fits (Fig. 11). As expected from the different behavior of the two absorption components with increasing energy levels, the derived rotation temperatures using the 4 lower energy lines of the $\sim-7 \mathrm{~km} \mathrm{~s}^{-1}$ and $\sim+5 \mathrm{~km} \mathrm{~s}^{-1}$ are $\sim 163 \pm 20 \mathrm{~K}$ and $\sim 103 \pm 10 \mathrm{~K}$, respectively. As a comparison, rotational temperatures measured at submm wavelength from $\mathrm{CH}_{3} \mathrm{OH}$ emission lines toward source $\mathrm{n}$ are around $200 \mathrm{~K}$ (Beuther et al. 2005b). Considering that these measurements are conducted with different molecules and very different observational techniques, the overall range of similar temperatures derived at mid-infrared and submm wavelengths is reassuring for the complementarity of such multi-wavelengths observations.

Fitting only the 4 lower-energy lines, the column density of ${ }^{13} \mathrm{CO}$ divided by the partition function $Q(T)$ results in $N_{\text {tot }}\left({ }^{13} \mathrm{CO}\right) / \mathrm{Q}(\mathrm{T}) \approx 1.0 \times 10^{15} \mathrm{~cm}^{-2}$ and $N_{\text {tot }}(13 \mathrm{CO}) / \mathrm{Q}(\mathrm{T}) \approx$ $2.5 \times 10^{15} \mathrm{~cm}^{-2}$ for the $\sim-7 \mathrm{~km} \mathrm{~s}^{-1}$ and $\sim+5 \mathrm{~km} \mathrm{~s}^{-1}$ components, respectively. Approximating again the partition function by $Q(T) \sim k t /(h B)$ (Sect. 3.1.1) we have $Q(163 \mathrm{~K}) \approx 62$ and $Q(103 \mathrm{~K}) \approx 39$, and using the ${ }^{12} \mathrm{CO}$ to ${ }^{13} \mathrm{CO}$ isotopologic ratio of 69 (e.g., Sheffer et al. 2007), the derived total

\footnotetext{
1 The equivalent width $A$ is measured by simultaneous Gaussian fits to both absorption features.
}

CO column densities toward source $\mathrm{n}$ are $\approx 4.3 \times 10^{18} \mathrm{~cm}^{-2}$ and $\approx 6.7 \times 10^{18} \mathrm{~cm}^{-2}$, of the same order as for the BN object. With the CO-to- $\mathrm{H}_{2}$ conversion factor of $8 \times 10^{-5}$, the corresponding $\mathrm{H}_{2}$ column densities are $5.3 \times 10^{22} \mathrm{~cm}^{-2}$ and $8.4 \times 10^{22} \mathrm{~cm}^{-2}$. Adding these two column density values, the total $\mathrm{H}_{2}$ column density traced toward source $\mathrm{n}$ by this near-infrared observations is $\sim 1.4 \times 10^{23} \mathrm{~cm}^{-2}$, more than an order of magnitude below the $\mathrm{H}_{2}$ column densities derived with the Submillimeter Array at $865 \mu \mathrm{m}\left(\sim 6 \times 10^{24} \mathrm{~cm}^{-2}\right.$, Beuther et al. 2004). Similar to the BN-case discussed in Sect. 3.1.1, this shows that the nearinfrared data mainly trace the warmer gas whereas the (sub)mm continuum observations trace warm and cold gas components.

\subsubsection{The Pfund $\beta$ line}

The Pfund $\beta$ line is also detected toward source $\mathrm{n}$ (Fig. 12), and we can fit a Gaussian to the recombination line with FWHM of $\Delta v \approx 24.3 \mathrm{~km} \mathrm{~s}^{-1}$, a width down to 0 intensity of $\approx 70 \mathrm{~km} \mathrm{~s}^{-1}$ (from $\sim-35$ to $\sim+35 \mathrm{~km} \mathrm{~s}^{-1}$ ) and a central velocity of $\sim 0 \mathrm{~km} \mathrm{~s}^{-1}$. The line is covered by 2 CRIRES chips, and while the general Pfund $\beta$ line shape is the same for both chips, the central dip at $\sim 0 \mathrm{~km} \mathrm{~s}^{-1}$ cannot independently be reproduced. Therefore, the dip is likely only an artifact due to insufficient signal to noise.

The thermal line width of a $10^{4} \mathrm{~K}$ hydrogen gas is $\sim 21.4 \mathrm{~km} \mathrm{~s}^{-1}$. Convolving that with the $6 \mathrm{~km} \mathrm{~s}^{-1}$ spectral resolution, the observable thermal line width should be $\sim 22.2 \mathrm{~km} \mathrm{~s}^{-1}$. Therefore, the measured line width does not exceed much the thermal line width of an HII region. Hence, the Pfund $\beta$ emission from source $n$ does not exhibit strong signatures from a wind but is rather consistent with a thermal HII region. We also cannot exclude that the Pfund $\beta$ emission toward source $\mathrm{n}$ is contaminated by more broadly distributed Orion nebula emission.

\section{3. $I R C 3$}

As outlined in the Introduction, in contrast to the $\mathrm{BN}$ object and source $n$, the source IRC3 is unlikely to be a YSO, and it is not prominent in any typical hot core tracer (e.g., Blake et al. 1996; Beuther et al. 2005b). The source rather resembles a more extended dust density enhancement that reprocesses light from other sources, potentially IRC2. Figure 13 presents a part of the $2 \mathrm{D}$ slit spectrum clearly showing the extended nature of the $\mathrm{CO}$ emission toward IRC3 in contrast to the point-like continuum structure from source $n$. The continuum emission from IRC3 is very weak compared to that from source n.

Figure 14 shows the $\mathrm{CO}$ lines extracted as an average spectrum of length 5.16" toward IRC3. While we again see a broad absorption feature against the weaker background extending from $\sim-5 \mathrm{~km} \mathrm{~s}^{-1}$ out to the telluric contamination at about $-40 \mathrm{~km} \mathrm{~s}^{-1}$, IRC3 shows a broad and strong emission feature peaking at $\sim 2 \mathrm{~km} \mathrm{~s}^{-1}$. Since this emission is extended over several arcseconds, Fig. 15 presents the position velocity diagrams of selected ${ }^{12} \mathrm{CO}$ and all ${ }^{13} \mathrm{CO}$ lines. In contrast to the $\mathrm{BN}$ object where the extended $\mathrm{CO}$ emission is just around the rest velocity of the star (Fig. 6), here we see a clear trend of increasing velocity with increasing distance from the continuum peak (the so-called Hubble law of outflows). Furthermore, in particular the ${ }^{12} \mathrm{CO}$ position velocity diagrams show a twofold structure with one velocity increase to values $>10 \mathrm{~km} \mathrm{~s}^{-1}$ at offsets of $\sim-1.8^{\prime \prime}$ and another increase to similar velocities at $\sim-3.3^{\prime \prime}$ offset from the main continuum peak. To guide the eye, these wedge-like structures are sketched in the top-right panel of Fig. 15). Such multiple wedge position velocity structures are 


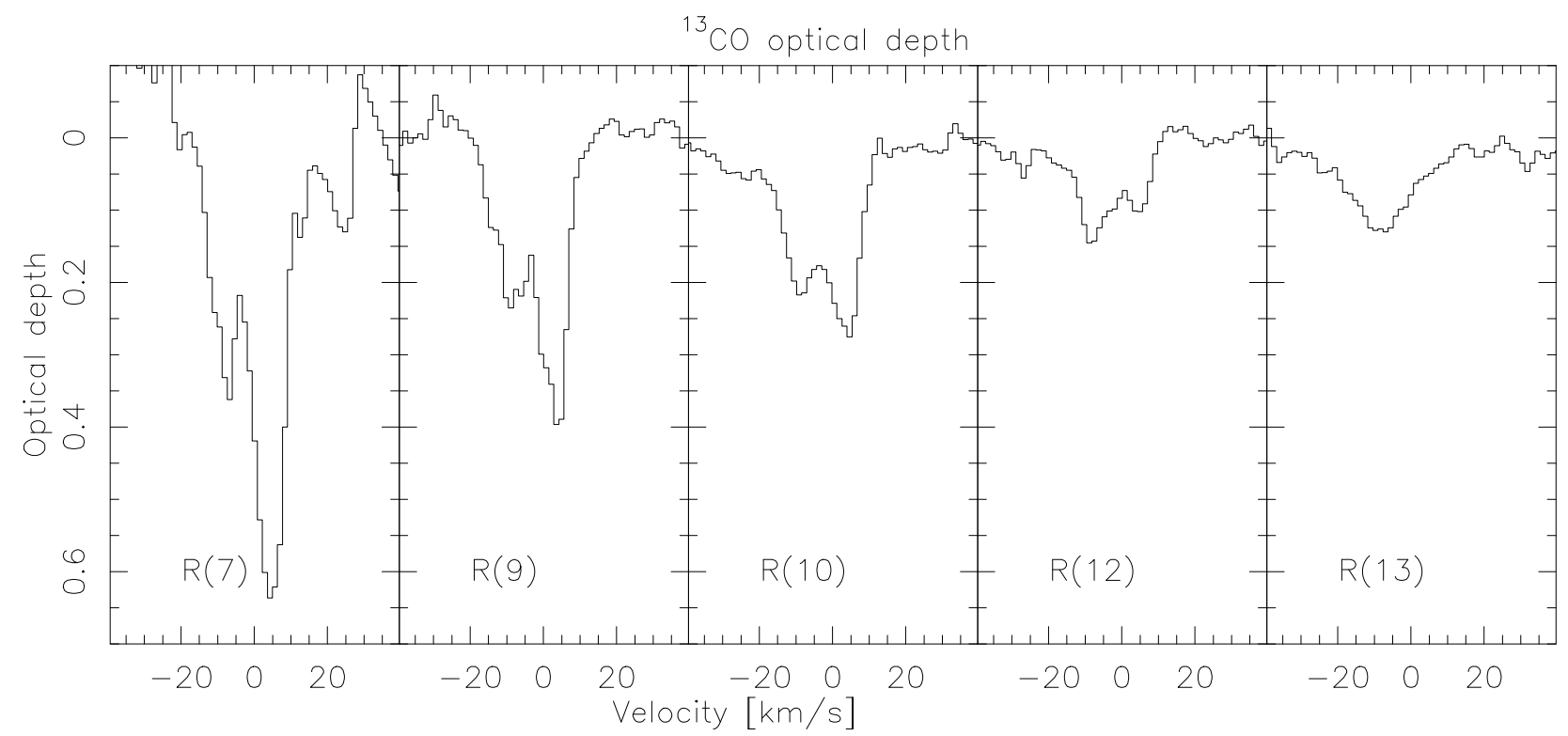

Fig. 10. ${ }^{13} \mathrm{CO}$ optical depths toward source $\mathrm{n}$. The absorption feature at $\sim 25 \mathrm{~km} \mathrm{~s}^{-1}$ of the $\mathrm{R}(7)$ line is likely an artifact due to telluric lines.
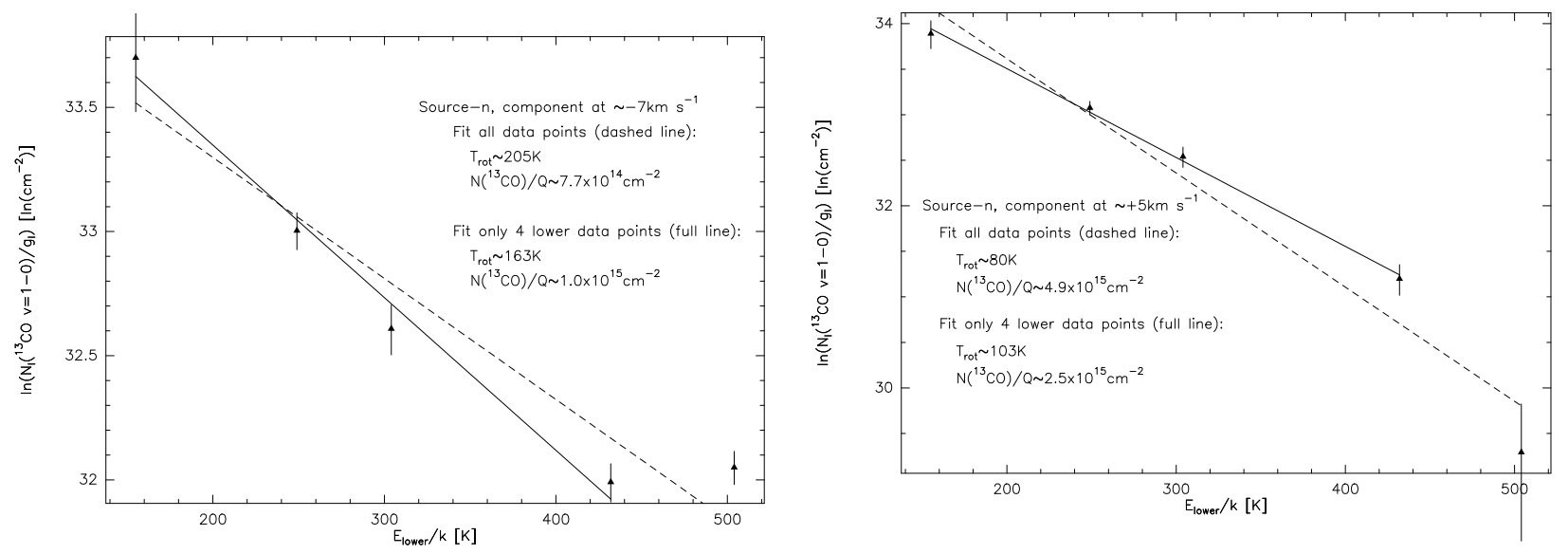

Fig. 11. Boltzmann plots for the ${ }^{13} \mathrm{CO} v=1-0$ lines toward source $\mathrm{n}$ for the $\sim-7 \mathrm{~km} \mathrm{~s}^{-1}$ and the $\sim+5 \mathrm{~km} \mathrm{~s}^{-1}$ components in the left and right panels, respectively. The $x$-axis shows the lower-level energies of the transitions and the $y$-axis presents the natural logarithm of the corresponding column densities divided by their statistical weights. In both panels, the dashed line shows a fit to all five data points, whereas the full line presents a fit to only the four lower transitions.

what jet-bow-shock entrainment models for molecular outflows predict (e.g., Arce et al. 2007). Furthermore, similar to source n, the $\mathrm{CO}$ absorption shows also for IRC3 the extended winglike features toward the most blue-shifted absorption. Similar to the pv-diagrams, where we see acceleration of the gas with distance from the source, these wing-like absorption can also be interpreted in the framework of accelerated winds (see also Sect. 3.2.1 or Lamers \& Cassinelli 1999). In summary, IRC3 is not only a dust density enhancement scattering the light from another source, it may also be part of the famous outflow emanating from the Orion-KL region. Based on these data, we cannot clearly define the driving source of the $\mathrm{CO}$ outflow feature. While the continuum peak of IRC 3 could be driving an outflow, the $\mathrm{CO}$ emission may also be part of the large-scale outflow where different driving sources have been proposed for in the literature, e.g., source I, SMA1, or the disintegration of a bound system once containing sources I, n and the BN-object (e.g., Menten \& Reid 1995; Gómez et al. 2005; Beuther \& Nissen 2008; Zapata et al. 2009). While the position velocity structure of the ${ }^{13} \mathrm{CO}$ lines largely resembles that of the ${ }^{12} \mathrm{CO}$ lines

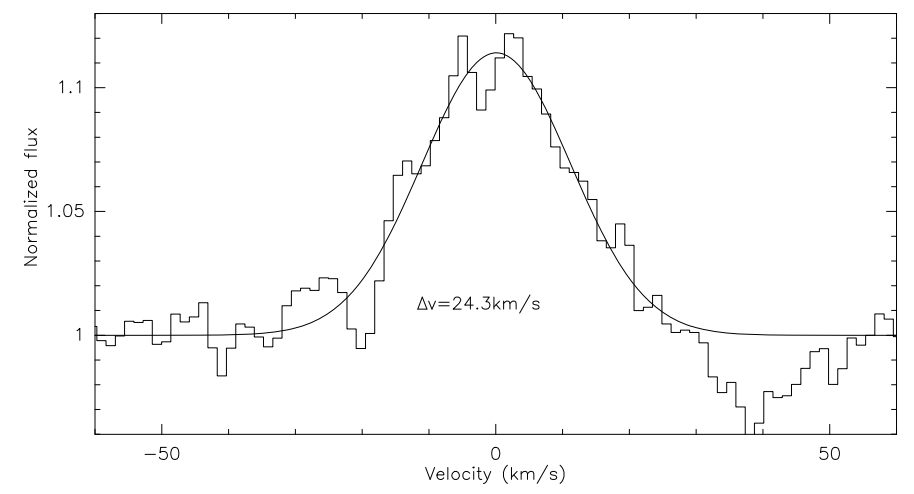

Fig. 12. Hydrogen recombination Pfund $\beta$ line of source $\mathrm{n}$.

(Fig. 15), it is interesting to note that the strongest ${ }^{13} \mathrm{CO}$ feature is at higher velocities and relatively distant from the continuum peak (offset $\sim-2.8^{\prime \prime}$ and velocity $\sim+7 \mathrm{~km} \mathrm{~s}^{-1}$. This difference is likely caused by the varying optical depth of the ${ }^{12} \mathrm{CO}$ 


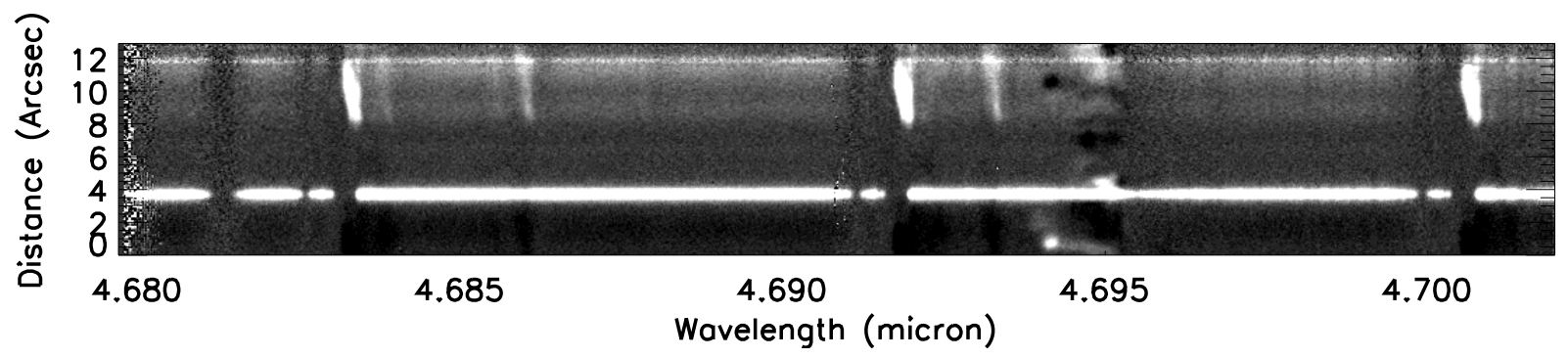

Fig. 13. Original data from the slit covering source $n$ and IRC3. Source $\mathrm{n}$ is the bright continuum source at offset $\sim 4^{\prime \prime}$ whereas IRC 3 exhibits the extended CO emission around offset $\sim 10^{\prime \prime}$.

and ${ }^{13} \mathrm{CO}$ transitions. Since the velocity of the emission feature is very close to the $v_{\mathrm{lsr}}$ of the cloud (typically between 5 and $9 \mathrm{~km} \mathrm{~s}^{-1}$, e.g., Comito et al. 2005), self-absorption of the most abundant ${ }^{12} \mathrm{CO}$ isotopologue may veil this feature, whereas the less abundant ${ }^{13} \mathrm{CO}$ can be utilised to penetrate more deeply into the cloud and hence detect the emission better.

\section{Discussion and conclusion}

High spectral resolution mid-infrared observations allow us to infer several important characteristics of some of the major sources within the Orion-KL region. The fundamental CO lines are largely dominated by absorption from the individual YSO envelopes and their surrounding gas cloud. These absorption features are blue- and red-shifted with respect to the $v_{\text {lsr }}$ of the molecular cloud indicating that outflowing and inflowing gas are simultaneously present toward our target sources. However, we also identify interesting emission features. Together they confirm the youth of the sources where infall and likely accretion are still ongoing.

BN object: for the BN object, our data confirm, at double the spectral resolution, several of the assessments conducted already by Scoville et al. (1983). However, also discrepancies arise. For example, an absorption feature reported previously around $+30 \mathrm{~km} \mathrm{~s}^{-1}$ is not found in the new data, indicating either transient components or poor signal-to-noise in the older data. From a Boltzmann analysis, the rotational temperature is around $112 \mathrm{~K}$, and we derive CO column densities of several times $10^{18} \mathrm{~cm}^{-2}$, well in agreement with the older results based on ${ }^{12} \mathrm{CO} v=2-0$ by Scoville et al. (1983). Using standard COto- $\mathrm{H}_{2}$ conversion factors, these column densities are about an order of magnitude below $\mathrm{H}_{2}$ column density estimates based on mm continuum emission. As discussed in Sect. 3.1.1, while systematics may account for some of this discrepancy, the main difference is that the mm continuum emission is sensitive to cold and warm dust, whereas the near-infrared absorption lines trace only the warm gas components.

We also identify extended $\mathrm{CO}$ emission, likely emanating from the close environment of BN. While the absorption stems from warm gas with temperatures on the order of $100 \mathrm{~K}$, getting the ro-vibrationally lines in emission implies already much higher temperatures for the corresponding gas components. The velocity range of the $\mathrm{CO}$ emission between 15 and $30 \mathrm{~km} \mathrm{~s}^{-1}$ encompasses the previously inferred velocity of the BN object of $\sim 21 \mathrm{~km} \mathrm{~s}^{-1}$. Following Scoville et al. (1983), who covered more J-transitions (fundamental and overtone emission) than we do, the rotational temperature of the compact emitting gas is around $600 \mathrm{~K}$, significantly higher than the above derived rotational temperature of the absorbing envelope gas. As outlined

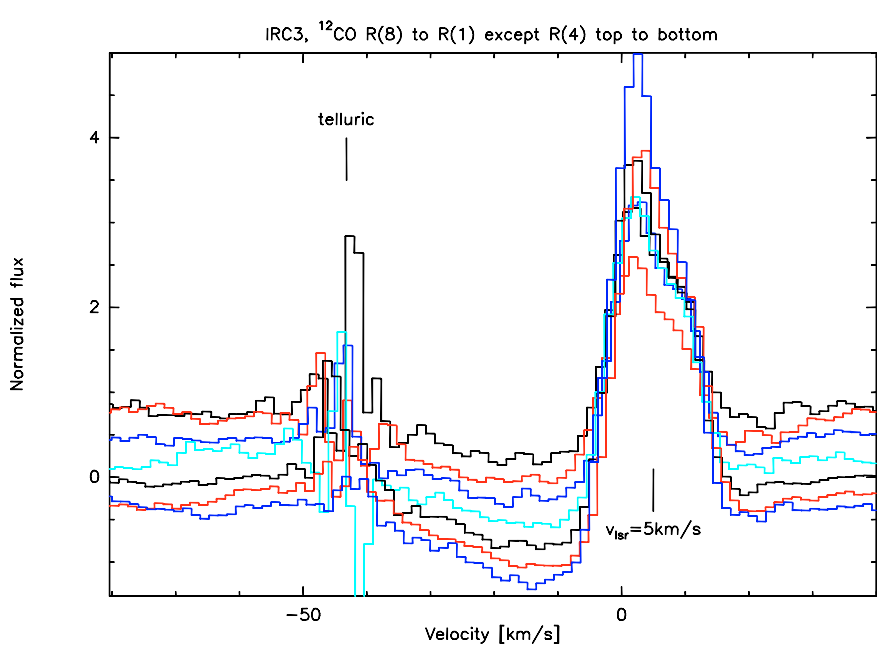

Fig. 14. The ${ }^{12} \mathrm{CO} \mathrm{R}(8)$ to $\mathrm{R}(1)$ lines (except of the $\mathrm{R}(4)$ line from top to bottom toward IRC3. Since the emission is extended, this spectrum is an average over 5.16" along the slit. Telluric and $v_{\text {lsr }}$ velocities are marked.

in Sect. 3.1.1, high critical densities are required to produce this line emission by pure thermal collisional excitation, and other processes like UV fluorescence or resonance scattering from infrared emission may also contribute to the emission lines. Scoville et al. (1983) infer an approximate size for that emission of $\sim 20 \mathrm{AU}$, whereas we now resolve the emission extending to $\sim \pm 4^{\prime \prime}$, corresponding to a diameter of $\sim 3300$ AU. This discrepancy can be explained by the wide dynamical range between the compact emission close to the source itself and the comparably very weak more extended emission. The small size found by Scoville et al. (1983) can be attributed to the strong compact emission which also emits in the overtone bands, whereas our more sensitive new data also detect the weaker extended features due to the high dynamic range available with CRIRES. While the absolute ratio of the peak emission (CO plus continuum) to the extended $\mathrm{CO}$ emission is $\sim 210$, the ratio of the continuum subtracted $\mathrm{CO}$ emission toward $\mathrm{BN}$ compared to the extended $\mathrm{CO}$ is still 70. Scoville et al. (1983) were not sensitive enough to detect this faint extended emission compared to the strong central source. Although at the spectral resolution limit of our observations, the measured line width increase of the ${ }^{13} \mathrm{CO}$ emission feature with increasing excitation temperature is consistent with a disk origin of the $\mathrm{CO}$ emission. Therefore, these parameters make the $\mathrm{CO}$ emitting gas potentially to be associated with a disk around the BN object (see also Jiang et al. 2005). Further supporting the disk-outflow interpretation for the BN object are the Pfund $\beta$ hydrogen recombination line data which show broad 


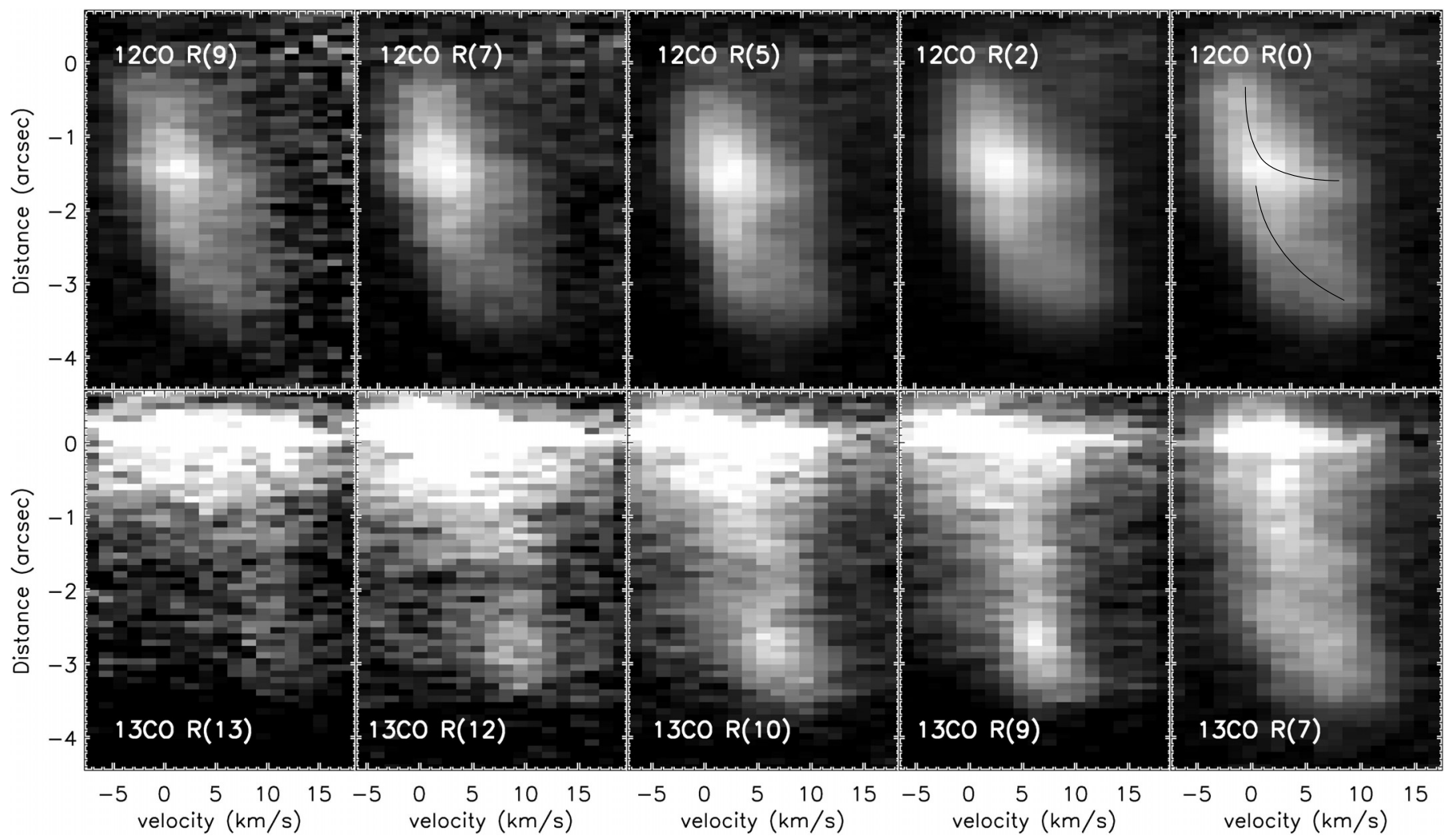

Fig. 15. Position velocity cuts along the slit axis for IRC3 with a PA of 110 degrees east of north. The top row shows diagrams for selected ${ }^{12} \mathrm{CO}$ lines, and the bottom row presents the ${ }^{13} \mathrm{CO}$ data. For ${ }^{13} \mathrm{CO}$ the scaling is adapted to highlight the weaker extended emission in contrast to the continuum at offset $0^{\prime \prime}$. The wedge-like outflow structures are sketched in the top-right panel.

high-velocity line wings consistent with the decelerating outflow scenario proposed by Scoville et al. (1983).

Source n: while bright sources like the BN object were already feasible to be observed a while ago (e.g., Scoville et al. 1983) weaker sources like source $\mathrm{n}$ or IRC3 were only possible to observe with reasonable high-spectral-resolution spectroscopy since the advent of recent instruments like CRIRES on the VLT. The general picture for source $\mathrm{n}$ is relatively similar. A single broad absorption feature extending approximately to $-65 \mathrm{~km} \mathrm{~s}^{-1}$ traces mainly the molecular outflow whereas red-shifted emission likely stems from an inner infalling and accreting envelope/disk. This picture is consistent with disk/outflow proposals for this source deduced from $\mathrm{cm}$ and mid-infrared wavelength imaging projects (e.g., Menten \& Reid 1995; Greenhill et al. 2004; Shuping et al. 2004). The clearly resolved doublepeaked ${ }^{13} \mathrm{CO}$ structure allows to conduct the Boltzmann analysis for both components. We find that the colder component has higher $\mathrm{H}_{2}$ column densities $\left(\sim 103 \mathrm{~K}\right.$ and $\left.\sim 8 \times 10^{22} \mathrm{~cm}^{-2}\right)$ compared with the second warmer component $(\sim 163 \mathrm{~K}$ and $\left.\sim 5 \times 10^{22} \mathrm{~cm}^{-2}\right)$. As discussed above for the BN object, differences between the infrared $\mathrm{CO}$ derived column densities and those estimated from $\mathrm{mm}$ wavelengths observations may arise because both tracers are sensitive to gas (and dust) at different temperatures. In contrast to the $\mathrm{BN}$ object, the Pfund $\beta$ emission from source $\mathrm{n}$ is consistent with a thermal HII region without a strong wind component to the line shape.

IRC3: the observational signatures from the dust density enhancement IRC3 are very different compared to the two previously discussed sources. The continuum from IRC3 is much weaker, nevertheless we detect $\mathrm{CO}$ absorption between $\sim-5$ and $\sim-40 \mathrm{~km} \mathrm{~s}^{-1}$. However, more importantly, we clearly detect extended $\mathrm{CO}$ and ${ }^{13} \mathrm{CO}$ emission over scales of $\sim 4^{\prime \prime}$. This extended $\mathrm{CO}$ emission shows a multiple wedge-like velocity structure consistent with jet-entrainment models of molecular outflows. Hence IRC3 may be not merely a dust density enhancement, but it may be part of the famous outflow from the Orion-KL region.

Limitations and future: one shortcoming of the data is that for our primary targets, the BN object and source n, we could not spatially resolve the inner region of the emission as done in the lower-mass case presented in Goto et al. (2006). The reasons may be different for the two sources. Source $n$ is probably still too young and too deeply embedded so that the envelope overwhelms any emission from the embedded disk itself. The BN object has the advantage that its velocity of rest is offset from that of the cloud by more than $10 \mathrm{~km}^{-1}$, and hence absorption could be less of a problem for such kind of source. However, $\mathrm{BN}$ is likely significantly more evolved, and the continuum-to-line ratio is so high that we cannot reasonably filter out the continuum emission. Hence, we cannot well study the inner region of the proposed disk. Nevertheless, observing higher J-transitions as well as the $\mathrm{CO}$ overtone emission with todays higher sensitivity compared to the Scoville et al. (1983) observations will likely constrain the proposed disk structure in more detail.

How to proceed now if one wants to do similar-type fundamental CO line studies of disks in high-mass star formation? On the one hand, it is important to not select too young sources because their envelopes will likely almost always "destroy" the emission signatures. There may exist exceptions where one 
views straight through the outflow cavity face-on toward the disk. On the other hand, for more evolved regions, the continuum emission can be very strong or maybe the remaining disk size can be reduced again making the spatial resolution a problem. Therefore, in addition to very careful target selections, adding AO to achieve the best spatial resolution will be a crucial element for such kind of studies in the coming years. Furthermore, in particular for the mostly saturated ${ }^{12} \mathrm{CO}$ lines, it will be important to extend the spectral coverage to also observe higher excited $\mathrm{CO}$ lines, that will likely not saturate anymore, as well as $\mathrm{CO}$ overtone emission. This will allow us to better assess the hotter gas components and hence to conduct a more detailed analysis of the ${ }^{12} \mathrm{CO}$ data themselves. On longer time-scales, the ELT with its proposed mid-infrared instrument METIS promises orders of magnitude progress in this field based on its superior sensitivity and spatial resolution. With this instrument, we will be truly capable to resolve the gas signatures of accretion disks around (high-mass) YSOs.

Acknowledgements. We like to thank a lot the anonymous referee as well as the Editor Malcolm Walmsley for thorough reviews which helped improving the paper. H.B. acknowledges financial support by the Emmy-Noether-Program of the Deutsche Forschungsgemeinschaft (DFG, grant BE2578).

\section{References}

Arce, H. G., Shepherd, D., Gueth, F., et al. 2007, in Protostars and Planets V, ed. B. Reipurth, D. Jewitt, \& K. Keil, 245

Bally, J. 2008, in Massive Star Formation: Observations Confront Theory, ed. H. Beuther, H. Linz, \& T. Henning, ASP Conf. Ser., 387, 158

Becklin, E. E., \& Neugebauer, G. 1967, ApJ, 147, 799

Beltrán, M. T., Cesaroni, R., Codella, C., et al. 2006, Nature, 443, 427

Beuther, H., \& Nissen, H. D. 2008, ApJ, 679, L121

Beuther, H., \& Walsh, A. J. 2008, ApJ, 673, L55

Beuther, H., Schilke, P., Menten, K. M., et al. 2002a, ApJ, 566, 945

Beuther, H., Schilke, P., Sridharan, T. K., et al. 2002b, A\&A, 383, 892

Beuther, H., Zhang, Q., Greenhill, L. J., et al. 2004, ApJ, 616, L31

Beuther, H., Schilke, P., Menten, K. M., et al. 2005a, ApJ, 633, 535

Beuther, H., Zhang, Q., Greenhill, L. J., et al. 2005b, ApJ, 632, 355

Blake, G. A., \& Boogert, A. C. A. 2004, ApJ, 606, L73

Blake, G. A., Mundy, L. G., Carlstrom, J. E., et al. 1996, ApJ, 472, L49

Brittain, S. D., Najita, J. R., \& Carr, J. S. 2009, ApJ, 702, 85

Cesaroni, R., Neri, R., Olmi, L., et al. 2005, A\&A, 434, 1039

Cesaroni, R., Galli, D., Lodato, G., Walmsley, C. M., \& Zhang, Q. 2007, in

Protostars and Planets V, ed. B. Reipurth, D. Jewitt, \& K. Keil, 197
Chernin, L. M., \& Wright, M. C. H. 1996, ApJ, 467, 676

Comito, C., Schilke, P., Phillips, T. G., et al. 2005, ApJS, 156, 127

Dougados, C., Lena, P., Ridgway, S. T., Christou, J. C., \& Probst, R. G. 1993, ApJ, 406, 112

Downes, D., Genzel, R., Becklin, E. E., \& Wynn-Williams, C. G. 1981, ApJ, 244,869

Draine, B. T., Dale, D. A., Bendo, G., et al. 2007, ApJ, 663, 866 Gómez, L., Rodríguez, L. F., Loinard, L., et al. 2005, ApJ, 635, 1166

Goto, M., Usuda, T., Dullemond, C. P., et al. 2006, ApJ, 652, 758

Greenhill, L. J., Gezari, D. Y., Danchi, W. C., et al. 2004, ApJ, 605, L57

Henning, T., Pfau, W., \& Altenhoff, W. J. 1990, A\&A, 227, 542

Henning, T., Michel, B., \& Stognienko, R. 1995, Planet. Space Sci., 43, 1333

Henning, T., Schreyer, K., Launhardt, R., \& Burkert, A. 2000, A\&A, 353, 211

Hildebrand, R. H. 1983, QJRAS, 24, 267

Jiang, Z., Tamura, M., Fukagawa, M., et al. 2005, Nature, 437, 112

Käufl, H.-U., Ballester, P., Biereichel, P., et al. 2004, in SPIE Conf. Ser., 5492, ed. A. F. M. Moorwood, \& M. Iye, 1218

Krumholz, M. R., Klein, R. I., \& McKee, C. F. 2007, ApJ, 656, 959

Krumholz, M. R., Klein, R. I., McKee, C. F., Offner, S. S. R., \& Cunningham, A. J. 2009, Science, 323, 754

Lamers, H. J. G. L. M., \& Cassinelli, J. P. 1999, Introduction to Stellar Winds (Cambridge University Press)

Lawrence, A., Warren, S. J., Almaini, O., et al. 2007, MNRAS, 379, 1599

Luhman, K. L. 2000, ApJ, 544, 1044

Menten, K. M., \& Reid, M. J. 1995, ApJ, 445, L157

Menten, K. M., Reid, M. J., Forbrich, J., \& Brunthaler, A. 2007, A\&A, 474, 515

Minchin, N. R., Hough, J. H., McCall, A., et al. 1991, MNRAS, 248, 715

Nissen, H. D., Gustafsson, M., Lemaire, J. L., et al. 2007, A\&A, 466, 949

Ossenkopf, V., \& Henning, T. 1994, A\&A, 291, 943

Plambeck, R. L., Wright, M. C. H., Mundy, L. G., \& Looney, L. W. 1995, ApJ, 455, L189

Pontoppidan, K. M., Boogert, A. C. A., Fraser, H. J., et al. 2008, ApJ, 678, 1005 Reipurth, B., Jewitt, D., \& Keil, K. 2007, Protostars and Planets V

Rothman, L., Jacquemart, D., Barbe, A., et al. 2005, J. Quant. Spec. Radiat. Transf., 96, 139

Ryde, N., \& Schöier, F. L. 2001, ApJ, 547, 384

Schreyer, K., Henning, T., van der Tak, F. F. S., Boonman, A. M. S., \& van Dishoeck, E. F. 2002, A\&A, 394, 561

Scoville, N., Kleinmann, S. G., Hall, D. N. B., \& Ridgway, S. T. 1983, ApJ, 275, 201

Scoville, N. Z., Carlstrom, J. E., Chandler, C. J., et al. 1993, PASP, 105, 1482

Sheffer, Y., Rogers, M., Federman, S. R., Lambert, D. L., \& Gredel, R. 2007, ApJ, 667, 1002

Shuping, R. Y., Morris, M., \& Bally, J. 2004, AJ, 128, 363

Tan, J. C. 2004, ApJ, 607, L47

van der Plas, G., van den Ancker, M. E., Acke, B., et al. 2009, A\&A, 500, 1137

Yorke, H. W., \& Sonnhalter, C. 2002, ApJ, 569, 846

Zapata, L. A., Schmid-Burgk, J., Ho, P. T. P., Rodríguez, L. F., \& Menten, K. M. 2009, ApJ, 704, L45

Zhang, Q., Hunter, T. R., Brand, J., et al. 2005, ApJ, 625, 864 